\title{
Oxidized CaMKII and 0-GIcNAcylation cause increased atrial fibrillation in diabetic mice by distinct mechanisms
}

\author{
Olurotimi O. Mesubi, ${ }^{1}$ Adam G. Rokita,, ${ }^{2,3}$ Neha Abrol, ${ }^{1}$ Yuejin Wu, ${ }^{1}$ Biyi Chen, ${ }^{2}$ Qinchuan Wang, ${ }^{1}$ Jonathan M. Granger, ${ }^{1}$ \\ Anthony Tucker-Bartley, ${ }^{1}$ Elizabeth D. Luczak, ${ }^{1}$ Kevin R. Murphy, ${ }^{1}$ Priya Umapathi, ${ }^{1}$ Partha S. Banerjee, ${ }^{4}$ Tatiana N. Boronina, ${ }^{4}$ \\ Robert N. Cole, ${ }^{4}$ Lars S. Maier, ${ }^{3}$ Xander H. Wehrens, ${ }^{5}$ Joel L. Pomerantz, ${ }^{4,6}$ Long-Sheng Song, ${ }^{2}$ Rexford S. Ahima, ${ }^{7}$ Cerald W. Hart, ${ }^{4}$ \\ Natasha E. Zachara, ${ }^{4}$ and Mark E. Anderson ${ }^{1,8,9}$ \\ 'Division of Cardiology, Department of Medicine, Johns Hopkins University School of Medicine, Baltimore, Maryland, USA. ²Division of Cardiovascular Medicine and Cardiovascular Research Center, Carver \\ College of Medicine, lowa City, lowa, USA. ${ }^{3}$ Department of Internal Medicine II, University Hospital Regensburg, Regensburg, Cermany. ${ }^{4}$ Department of Biological Chemistry, Johns Hopkins University School \\ of Medicine, Baltimore, Maryland, USA. ${ }^{5}$ Department of Molecular Physiology and Biophysics, Department of Medicine (Cardiology), Department of Pediatrics, and Center for Space Medicine, Baylor College \\ of Medicine, Houston, Texas, USA. Institute for Cell Engineering, ${ }^{7}$ Division of Endocrinology, Diabetes and Metabolism, Department of Medicine, ${ }^{8}$ Department of Medicine, and ${ }^{9}$ Department of Physiology \\ and Program in Cellular and Molecular Medicine, Johns Hopkins University School of Medicine, Baltimore, Maryland, USA.
}

\begin{abstract}
Diabetes mellitus (DM) and atrial fibrillation (AF) are major unsolved public health problems, and diabetes is an independent risk factor for AF. However, the mechanism(s) underlying this clinical association is unknown. ROS and protein 0 GIcNAcylation (OCN) are increased in diabetic hearts, and calmodulin kinase II (CaMKII) is a proarrhythmic signal that may be activated by ROS (oxidized CaMKII, ox-CaMKII) and OCN (OCN-CaMKII). We induced type 1 (T1D) and type 2 DM (T2D) in a portfolio of genetic mouse models capable of dissecting the role of ROS and OCN at CaMKII and global OCN in diabetic AF. Here, we showed that T1D and T2D significantly increased AF, and this increase required CaMKII and OCN. T1D and T2D both required ox-CaMKII to increase AF; however, we did not detect OGN-CaMKII or a role for OGN-CaMKII in diabetic AF. Collectively, our data affirm CaMKII as a critical proarrhythmic signal in diabetic AF and suggest ROS primarily promotes AF by ox-CaMKII, while OGN promotes AF by a CaMKII-independent mechanism(s). These results provide insights into the mechanisms for increased AF in DM and suggest potential benefits for future CaMKII and OCN targeted therapies.
\end{abstract}

\section{Introduction}

Atrial fibrillation (AF) and diabetes mellitus (DM) are major, unsolved public health problems (1-3). The burden of both conditions is projected to increase significantly in the United States and worldwide in the coming decades $(1,4-7)$. AF is the most common clinical arrhythmia (8), and it is associated with significant mortality and morbidity (9-11), such as stroke and heart failure. It is increasingly clear that DM, both type $1 \mathrm{DM}$ (T1D) and type $2 \mathrm{DM}$ (T2D), is an independent risk factor for AF (12-14), and the coexistence of AF and DM results in increased mortality, suffering, and cost (15-17). However, current therapies, such as antiarrhythmic drugs and catheter ablation $(18,19)$, are inadequate. Thus, improved understanding of the molecular mechanisms connecting DM and AF is an important goal for developing more effective therapies.

Conflict of interest: XHW is a cofounder and shareholder of ELEX Biotech, a company aiming to develop RyR2 inhibitor therapeutics. CWH receives a share of royalties, which are managed by Johns Hopkins University, generated from sales of the CTD 110.6 antibody. MEA has intellectual property rights regarding CaMKII inhibitors owned by Johns Hopkins University.

Copyright: (5) 2021, American Society for Clinical Investigation.

Submitted: June 16, 2017; Accepted: November 3, 2020; Published: January 19, 2021

Reference information: J Clin Invest. 2021;131(2):e95747.

https://doi.org/10.1172/JCI95747.
The multifunctional calcium and calmodulin-dependent protein kinase II (CaMKII) has emerged as a proarrhythmic signal in $\mathrm{AF}$ in the absence of DM (20-22). Our work and that of others suggests that ROS (23) and O-GlcNAcylation (OGN) (24) can activate CaMKII via modification of the CaMKII regulatory domain, leading to inappropriate $\mathrm{Ca}^{2+}$ leak from myocardial type 2 ryanodine receptors (RyR2), triggered action potentials, and arrhythmias (20, 24-27). Intriguingly, the ROS-modified (methionines 281/282; oxidized CaMKII, ox-CaMKII) and OGN-modified (serine 280; OGN-CaMKII) residues of the CaMKII regulatory domain are adjacent (Figure 1A), suggesting the hypothesis that CaMKII may integrate these signals - ROS and OGN, which are present in diabetic atrium - to favor AF. The role of these signals, and whether they operate independently or together, is untested in vivo. A recent study implicates OGN-CaMKII in increased ROS production and CaMKII signaling independent of ox-CaMKII in isolated ventricular myocytes in response to acute hyperglycemia (28). However, the potential proarrhythmic contributions of ox-CaMKII and OGN-CaMKII have not been directly determined in vivo in atria or in diabetic AF.

DM is characterized by hyperglycemia, elevated levels of ROS $(29,30)$, and increased OGN (31). OGN is an evolutionarily conserved nutrient- and stress-sensing posttranslational protein modification that occurs by covalent attachment of single $O$-linked $N$-acetylglucosamine $(O$-GlcNAc) residues to 
the amino acids serine and threonine, resulting in alteration of protein function akin to phosphorylation (32). This dynamic process is controlled exclusively by 2 enzymes: $O$-GlcNAc transferase (OGT), which catalyzes the addition of $O$-GlcNAc to target residues, and $O$-GlcNAcase (OGA), which catalyzes the removal of $O-G l c N A c(32,33)$. Atria from patients with diabetes have increased ROS (34) and OGN (35). Both ROS and OGN are associated with diabetic cardiomyopathy and $\operatorname{AF}(20,24,29,36-38)$, but it is uncertain whether these changes contribute to proarrhythmic molecular pathways favoring AF.

We reasoned that hyperglycemia secondary to DM is proarrhythmic and promotes AF through increased ROS and OGN upstream of CaMKII with consequent activation of RyR2 and potentially other targets, leading to increased proarrhythmic triggered activity (Figure 1B). We performed experiments to investigate the molecular mechanism(s) by which CaMKII integrates upstream signals from ROS and OGN to promote AF in DM to understand whether CaMKII activation by ROS and/or OGN are critical proarrhythmic mechanisms for diabetic AF in T1D and T2D. We induced T1D or T2D in a panel of mouse models in which ROS and OGN activation of CaMKII were selectively ablated, ROS and OGN upstream pathways were controlled, and convergent actions of CaMKII on RyR2 were prevented or mimicked. Our results establish CaMKII as a critical sensor of ROS and hyperglycemia and CaMKII activation of RyR2 as an important proarrhythmic pathway for $\mathrm{AF}$ in $\mathrm{DM}$. We found that $\mathrm{AF}$ in T1D and T2D required ox-CaMKII. In contrast, we found no evidence of OGN modification of CaMKII at S280 in diabetic mouse hearts, whereas loss of OGN-CaMKII by knockin replacement of the previously reported CaMKII OGN site (S280A) was not protective of AF induction in DM. However, we also found that transgenic myocardial overexpression of OGA protected against diabetic AF in both T1D and T2D, apparently by CaMKII-independent pathways.

\section{Results}

T1D and T2D mice have increased ROS and OGN. To test the hypothesis that CaMKII contributes to hyperglycemia-enhanced AF susceptibility, our first approach was to use suitable mouse models of T1D and T2D and determine myocardial levels of ROS and OGN. For the T1D mouse model, we injected mice with a single dose $(185 \mathrm{mg} / \mathrm{kg}$, i.p.) of streptozocin (STZ) (Figure 1C) (39). STZ-treated (T1D) mice had significantly higher blood glucose (Figure 1D and Supplemental Figure 1A; supplemental material available online with this article; https://doi.org/10.1172/JCI95747DS1) and reduced body weight (Figure 1E and Supplemental Figure 1B) compared with mice treated with a placebo (citrate buffer). There was no difference in heart weight indexed for body weight (Supplemental Figure 1C). T1D mice had slower heart rates (Supplemental Figure 1D), consistent with reports of defective heart rate in diabetic mice (39) and patients (40). Echocardiographic measurements showed impaired left ventricular (LV) systolic function in the T1D mice compared with nondiabetic littermate controls (Supplemental Figure 1E) without LV hypertrophy or dilation (Supplemental Figure 1, F and $\mathrm{H}$ ), findings similar to patients with diabetic cardiomyopathy (41). The T1D mice showed no evidence of ketoacidosis or renal failure (Supplemental Table 1). Thus, the T1D mice showed marked elevation in glucose, weight loss, and modest but consistent impairment in myocardial function, similar to previous reports of diabetic cardiomyopathy (42).
We considered the T1D model an ideal experimental platform to test the direct link between hyperglycemia and AF. However, the predominant form of DM in the human adult population affected by AF is T2D (43), a more complex disease characterized by hyperglycemia, impaired insulin secretion and insulin resistance (44). Thus, we next generated a T2D mouse model using 5- to 6-weekold male C57BL/6J mice fed a high-fat diet; 5 weeks after initiation of the high-fat diet, the mice received daily injections of low-dose STZ (40 mg/kg/day, i.p.) for 3 consecutive days (Figure $1 \mathrm{~F}$ ). The T2D mice had elevated blood glucose (Figure $1 G$ ); increased body weight, albeit with a slight decrease after STZ injection (Figure $1 \mathrm{H}$ and Supplemental Figure 2A); and no change in fasting insulin (Supplemental Figure 2B) but increased insulin resistance, determined by the homeostatic model assessment of insulin resistance (HOMA-IR) (45) (Supplemental Figure 2C), compared with untreated WT controls. Consistent with the defining features of T2D, our mice showed reduced glucose and insulin tolerance (Supplemental Figure 2, D and E) 2 weeks after low-dose STZ injection.

We next measured atrial ROS and OGN from whole-heart lysates from WT T1D, T2D, and nondiabetic control hearts. Consistent with prior animal $(24,29,30,36)$ and human data $(24,34-36)$, diabetic heart tissues showed increased ROS (Figure 2A) and OGN (Figure 2B) compared with WT nondiabetic controls. These foundational data suggested these models would be useful for testing the hypothesis that ox-CaMKII and OGN-CaMKII contributed to AF in T1D and T2D.

Increased AF susceptibility in T1D and T2D is CaMKII dependent. Excessive CaMKII activity is implicated in nondiabetic AF in patients (20-22), large animal models $(21,46)$, and mice $(20,21,47)$. More recently, OGN-CaMKII has been linked to ventricular arrhythmias in diabetic cardiomyopathy $(24,28)$. To test whether these diabetic mouse models are susceptible to increased AF, we performed rapid, right atrial burst pacing in anesthetized mice using an established protocol (Figure $3 \mathrm{~A})(20,21,48)$, with modification of the definition of AF in this study (see Methods). T1D mice had significantly higher AF compared with nondiabetic controls (Figure 3B). To further probe the effect of hyperglycemia on AF susceptibility, T1D mice were treated with insulin (delivered via osmotic pumps, LinBit) for 1 week after STZ injection. Insulin-treated T1D mice with rescue of hyperglycemia ( $<300 \mathrm{mg} / \mathrm{dL}$, Figure $3 \mathrm{C}$ ) had significantly reduced AF susceptibility compared with insulin-treated T1D mice with persistent hyperglycemia ( $>300 \mathrm{mg} / \mathrm{dL}$, Figure 3D). These findings, taken together with other reports $(13,14,49)$, confirmed that T1D is an AF risk factor in mice, similar to the situation in patients.

To test whether CaMKII contributes to hyperglycemia-enhanced AF susceptibility, we used mice with myocardial-targeted transgenic expression of AC3-I, a CaMKII inhibitory peptide (50). We found that AC3-I mice with T1D were protected from AF compared with WT nondiabetic controls (Figure 3B). Despite the reduction in AF, T1D AC3-I mice had similar increases in blood glucose as the WT T1D mice (Figure 1D). These findings supported the hypothesis that CaMKII is a proarrhythmic signal coupling DM to increased risk of AF. We next tested AF susceptibility in the T2D mouse model (Figure $1 \mathrm{~F}$ ). The T2D mice had significantly higher AF compared with nondiabetic controls (Figure 3E), similar to the findings in T1D mice and in humans with T1D and T2D (12-14). To determine whether CaMKII contributes to AF susceptibility in T2D, we performed right atrial burst pacing in AC3-I T2D mice. Similar to our findings in T1D, 
A

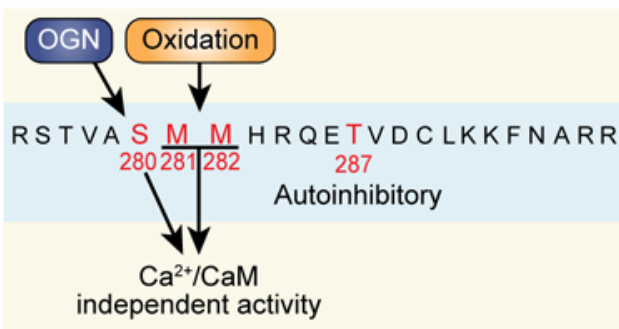

C
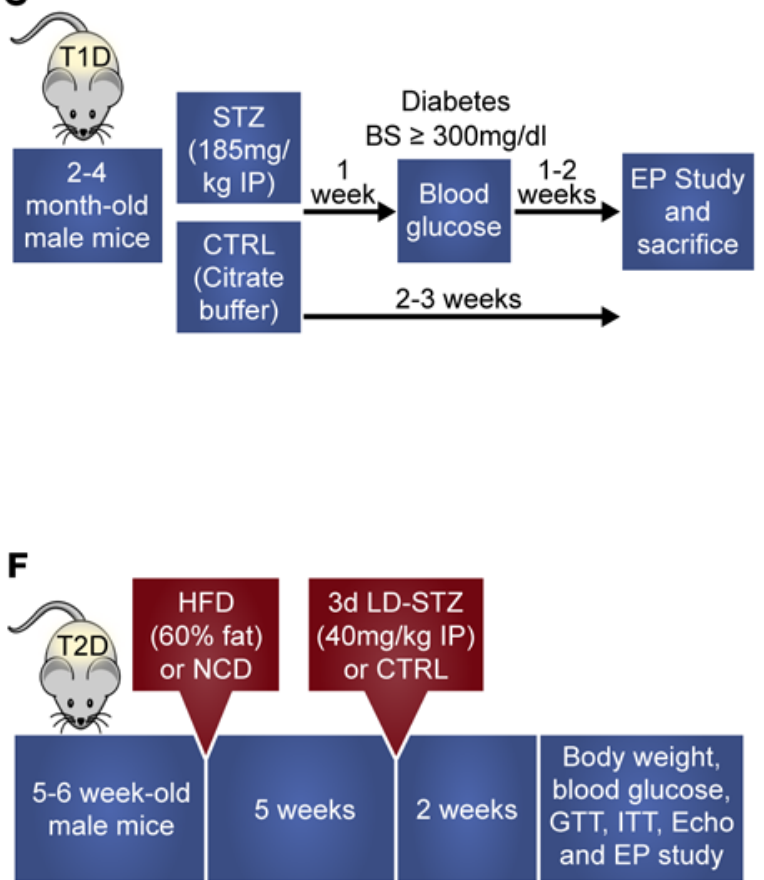

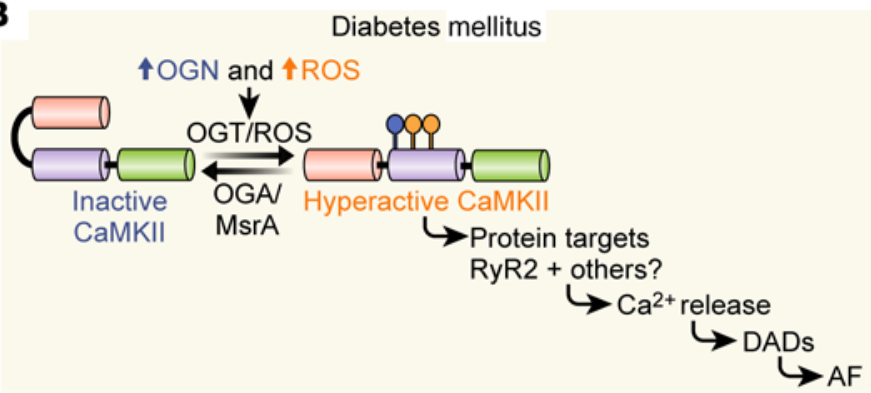

D
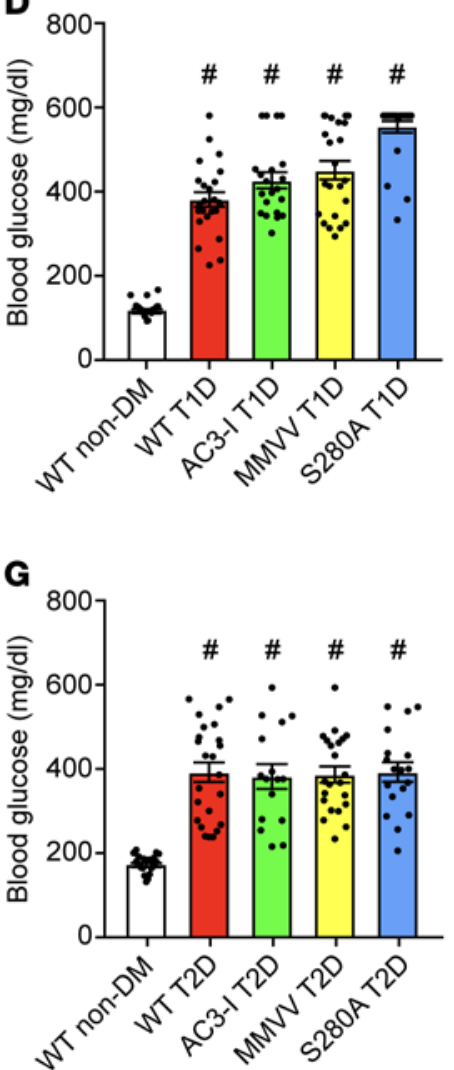

E

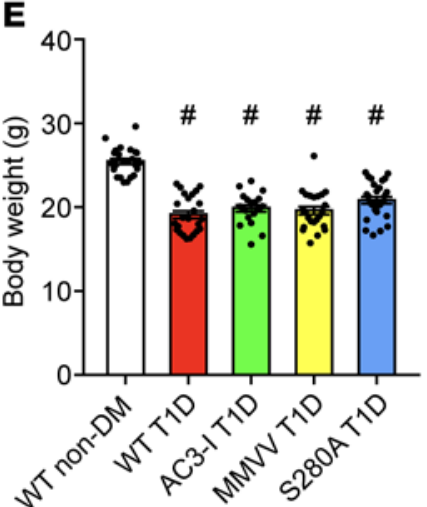

H

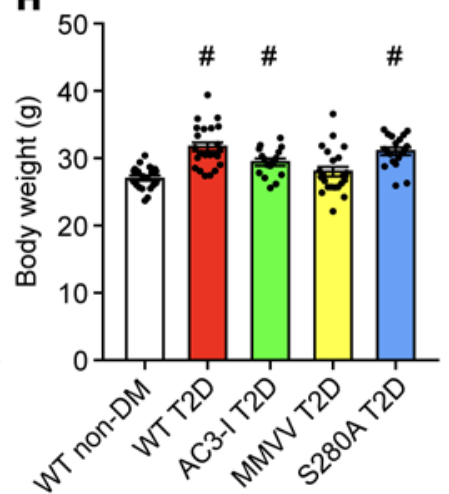

Figure 1. Proposed CaMKII posttranslational modifications and type 1 and type 2 diabetic models. (A) Oxidation at methionines $281 / 282$ and OCN at serine 280 are posttranslational modifications hypothesized to promote diabetic heart disease and arrhythmias. (B) Schematic representation of proposed hypothesis that excessive ROS and OGN in diabetes mellitus (DM) promotes AF through CaMKII-dependent signaling. (C) Schematic of diabetes induction and experimental protocol for T1D. Summary data for (D) blood glucose and (E) body weight in nondiabetic and T1D mice 2 weeks after STZ injection. (F) Schematic of DM induction and experimental protocol for T2D. Summary data for (C) blood glucose and (H) body weight in nondiabetic and T2D mice 2 weeks after LD-STZ injection. T1D, type 1 DM; T2D, type 2 DM; HFD, high-fat diet; LD-STZ, low-dose STZ; NCD, normal chow diet; OCN, O-GlcNAcylation; RyR2, ryanodine receptor type 2; STZ, streptozocin. Data are represented as mean \pm SEM. Statistical comparisons were performed using 1-way ANOVA with Tukey's multiple-comparison test (D, E, G and $\mathbf{H})\left(\# P<0.0001\right.$ vs. WT non-DM, ${ }^{*} P<0.05$ vs. WT non-DM).

AC3-I T2D mice were protected from AF (Figure 3E) compared with nondiabetic controls and had similar levels of hyperglycemia as WT T2D mice (Figure 1G). Taken together, these findings support the hypothesis that CaMKII is a proarrhythmic signal that is essential for increased risk of AF in validated mouse models of T1D and T2D.

Differential effect of MM281/282 and S280 on AF susceptibility in T1D and T2D. After initial activation, the CaMKII regulatory domain undergoes posttranslational modification that locks CaMKII into a constitutively active, $\mathrm{Ca}^{2+}$ - and calmodulin-independent conformation, and this promotes arrhythmias and cardiomyopathy $(51,52)$. ROS activates CaMKII by oxidizing a pair of methionines
(M281/282) (23), and OGN is reported to activate CaMKII by modifying serine 280 (S280) (24) (Figure 1A). The numbering is for CaMKII $\delta$, the most abundant CaMKII isoform in ventricular myocardium $(53,54)$. We used qRT-PCR to measure expression of each CaMKII isoform in human and mouse atrium. We found that, similar to the situation in the ventricle, CaMKII $\delta$ had the highest mRNA expression in human (Supplemental Figure 3A and Supplemental Table 2) and mouse atrium (Supplemental Figure 3B). In addition, the CaMKII isoform mRNA expression profile was unchanged in T1D and T2D atria compared with WT nondiabetic controls (Supplemental Figure 3, C and D). 
A
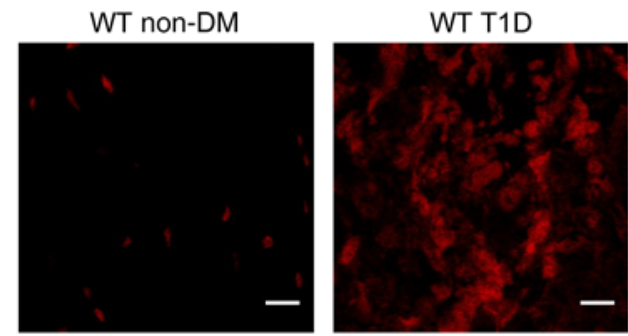

WT non-DM
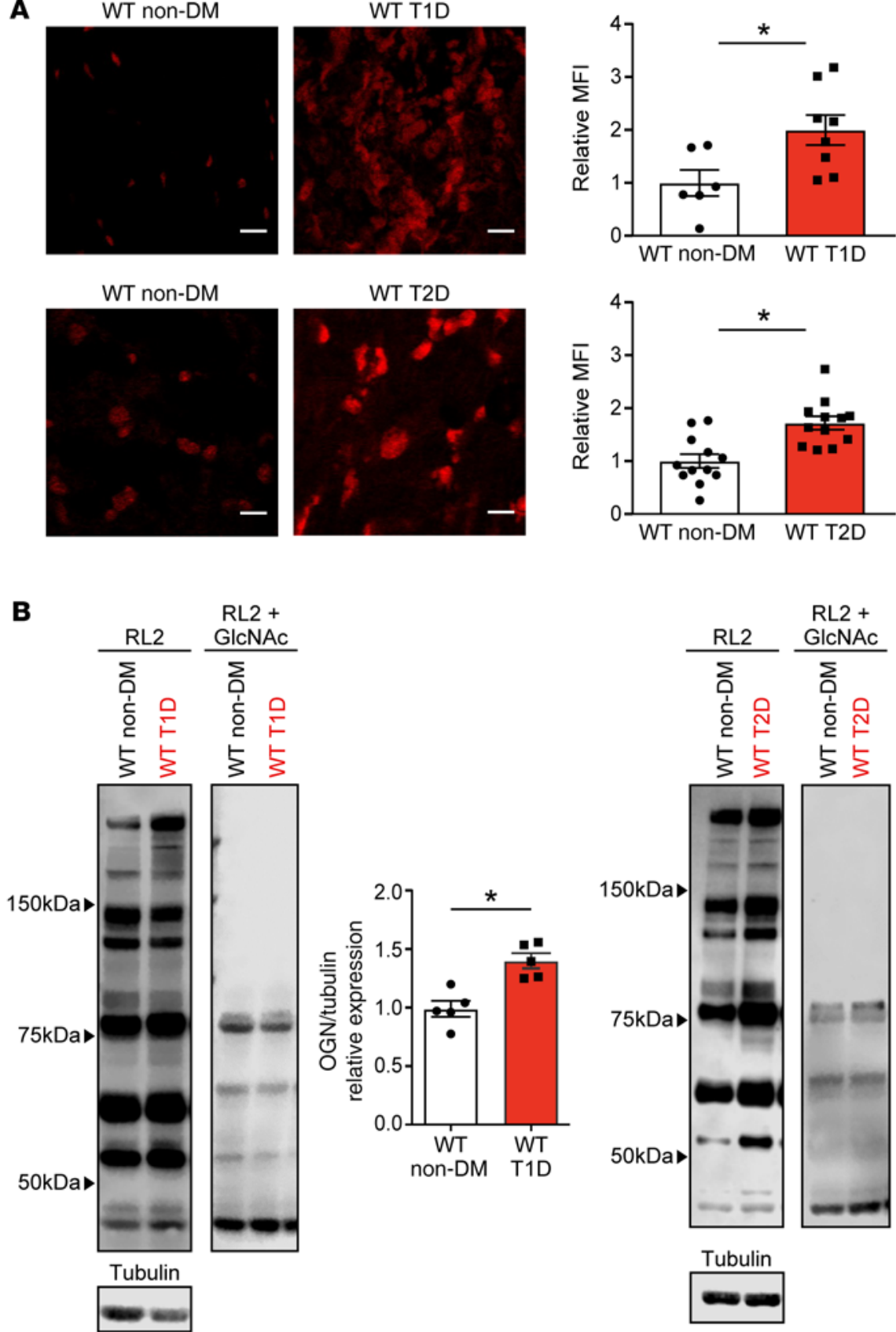

Figure 2. ROS and O-GIcNAcylation are elevated in type 1 and type 2 diabetic hearts. (A) Representative confocal images (original magnification, $\times 40$ ) and summary data for DHE fluorescence in mouse atrial tissue show increased ROS in T1D (top) and T2D (bottom). Scale bars: $10 \mu \mathrm{m}$ ( $n=6 \mathrm{WT}$ non-DM, $n=8 \mathrm{WT}$ T1D, $n=4$ WT non-DM, $n=4$ WT T2D) (B) Representative Western blots and summary data for total OGN modified protein levels (OGN monoclonal antibody - RL2) normalized to tubulin and

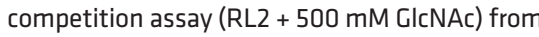
heart lysates, from T1D (left) and T2D (right) (n $=3-7 /$ group). OGN quantification excluded the noncompeted bands. DHE, dihydroethidium; $\mathrm{DM}$, diabetes mellitus; T1D, type $1 \mathrm{DM}$; T2D, type 2 DM; OGN, O-GIcNAcylation. Data are represented as mean \pm SEM. Statistical comparisons were performed using 2-tailed Student's $t$ test (A and $\mathbf{B})\left({ }^{*} P<0.05\right.$ vs. WT non-DM).
To determine the role of ox-CaMKII and OGN-CaMKII in hyperglycemia-primed AF, we used CaMKII $\delta$ genetically modified (knockin) mice. Our lab previously generated an ox-CaMKII-resistant-knockin (MMVV-knockin) mouse (39). These MMVV mice are notable for exhibiting resistance to sudden death due to severe bradycardia after myocardial infarction during T1D (39) and are protected from angiotensin II infusion-primed AF (20). These findings provided support for the role of ox-CaMKII as a pathological signal in atrial myocardium. OGN activation of CaMKII is described as a modification at S280 (24), so we developed an OGN-CaMKII-resistant (S280A) mouse based on this previous finding using CRISPR/ Cas9 (55-57) (Supplemental Figure 4A). S280A mice were born in Mendelian ratios, had normal morphology, and similar baseline LV fractional shortening (Supplemental Figure 4B) and LV mass
(Supplemental Figure 4C) compared with WT littermate controls. MMVV, S280A, and WT mice had similar resting heart rates (Supplemental Figure 4D) and total myocardial CaMKII expression (Supplemental Figure 4, E and F). We interpreted these findings, taken together with previous studies on MMVV mice (20,39), to suggest that the S280 and M281/282 sites on CaMKII $\delta$ are dispensable for normal development and basal function.

We induced T1D in MMVV and S280A mice and performed AF induction studies. T1D MMVV mice were resistant to AF, but T1D S280A mice showed similar AF susceptibility as T1D WT mice (Figure 3B). The WT and S280A T1D mice showed a modest but consistent decrement in LV fractional shortening compared with nondiabetic counterparts (Supplemental Figure 1, E and $\mathrm{H})$. In contrast, MMVV mice were resistant to T1D-induced 
A
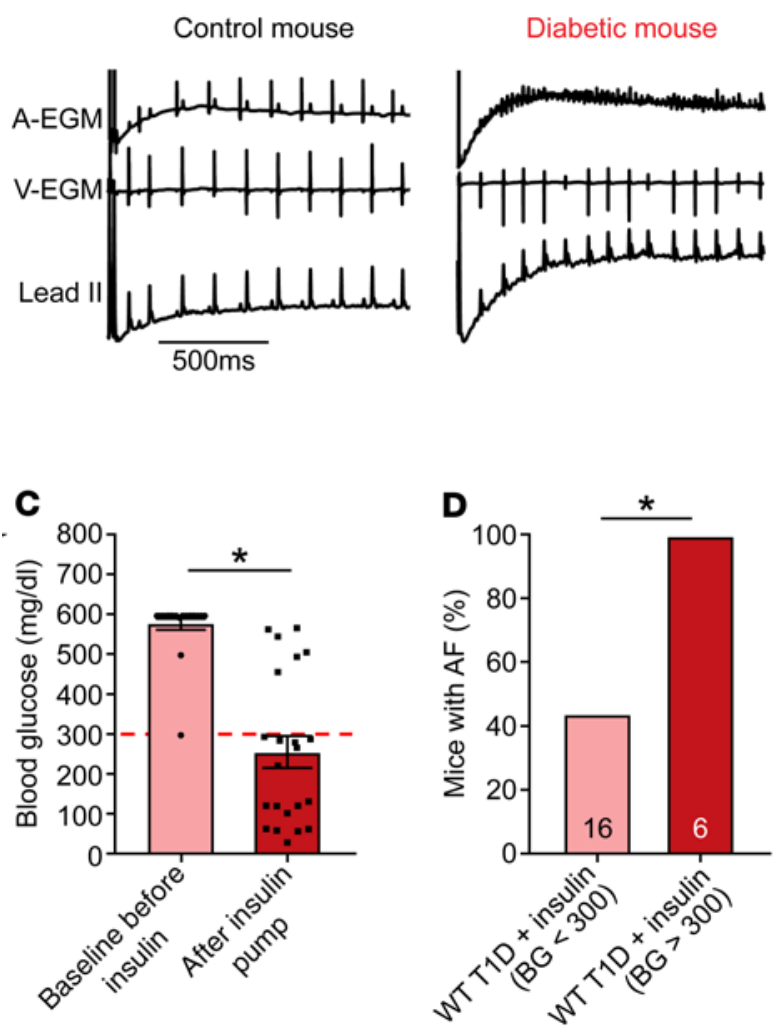

B

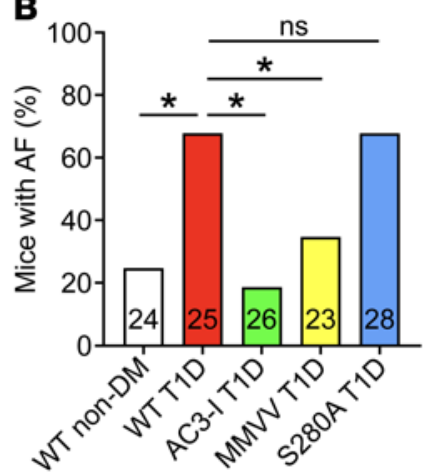

$\mathbf{E}$

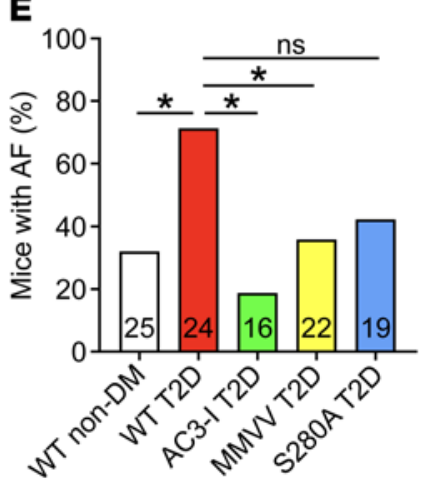

Figure 3. CaMKII promotes enhanced atrial fibrillation susceptibility in type 1 and type $\mathbf{2}$ diabetic mice. (A) Representative tracings of intracardiac (atrial, A-EGM; ventricular, V-EGM) and lead II surface electrocardiograms recorded immediately after rapid atrial burst pacing demonstrating normal sinus rhythm in a control non-DM WT mouse and irregular atrial and ventricular electrical impulses marking AF in a diabetic WT T1D mouse. (B) Marked AF susceptibility in WT T1D mice compared with WT non-DM mice. This is reversed in AC3-I and MMVV T1D mice, but not in S280A T1D mice. (C) Pre- and post-insulin pump (LinBit) implantation blood glucose levels 1 week after STZ treatment (pre-insulin pump) and 1 week after insulin pump implantation (post-insulin) ( $n=22$ ). (D) Insulin treatment prevents enhanced AF in WT T1D mice with blood glucose (BC) level less than $300 \mathrm{mg} / \mathrm{dL}$ on insulin treatment. (E) Increased AF susceptibility is present in WT T2D mice compared with nondiabetic controls; AC3-I and MMVV T2D mice are protected from enhanced AF, but there is no protection in S280A T2D mice. AF, atrial fibrillation; DM, diabetes mellitus; T1D, type $1 \mathrm{DM}$; T2D, type 2 DM; EGM, electrogram. Data are represented as percentage frequency distribution (B, D, and $\mathbf{E})$ and mean \pm SEM (C). The numerals in the bars represent the sample size in each group (B, D, and E). Statistical comparisons were performed using 2-tailed Fischer's exact test with Holm-Bonferroni correction for multiple comparisons (B, D, and E) and 2-tailed Student's $t$ test $(\mathbf{C})\left({ }^{*} P<0.05\right)$.

LV dysfunction (Supplemental Figure 4H). The T1D MMVV and S280A mice demonstrated similar increases in blood glucose and comparable body weight (Figure 1, D and E, and Supplemental Figure 4, I and J). These results suggested that ox-CaMKII at MM281/282, but not OGN-CaMKII at S280, contributed to T1D cardiomyopathy and AF. We next performed AF induction studies in T2D MMVV and S280A mice (Figure 3E). Similar to the findings in the T1D mice, T2D MMVV mice were resistant to AF, whereas T2D S280A mice showed similar AF susceptibility as T2D WT mice. We interpret these data to suggest that ox-CaMKII contributed to AF in both T1D and T2D mice. In contrast, the S280 site on CaMKII $\delta$ did not appear to play a critical proarrhythmic role in AF in T1D or T2D mice.

Hyperglycemia activates CaMKII in an MM281/282 ox-CaMKII- but not S280 OGN-CaMKII-dependent manner. The findings in the S280A mice in T1D were unexpected because of published evidence that OGN of CaMKII at S280 is required for hyperglycemia-induced ROS, SR $\mathrm{Ca}^{2+}$ leak, and triggered ventricular arrhythmias $(24,28)$. In order to determine whether increased OGN detected in diabetic mouse myocardium could directly increase
CaMKII activity, we used a fluorescent kinase reporter, recently validated in RPE-1 cells and mouse skeletal muscle $(58,59)$, based on work by Regot and colleagues (58). The fluorescent kinase translocation reporter (KTR) dynamically moves from the nucleus to the cytosol in response to kinase activation (Figure 4A), and the ratio of cytosol/nuclear distribution of the CaMKII-KTR fluorescent signal is a reliable measure of intracellular CaMKII activity (59). Isolated neonatal mouse cardiomyocytes transfected with CaMKII-KTR DNA plasmid 24 hours after isolation were cultured in low glucose (5.5 mM glucose) or high glucose (30 mM glucose) for 18 hours. Incubation with high glucose resulted in increased CaMKII activation compared with low-glucose conditions (Figure 4, B and C). There was no increase in CaMKII activation in neonatal cardiomyocytes incubated in hyperosmotic, euglycemic conditions (5.5 $\mathrm{mM}$ glucose $+24.5 \mathrm{mM}$ mannitol) compared with control glucose conditions. To determine whether CaMKII is critical for the KTR changes under hyperglycemic conditions, we co-incubated neonatal cardiomyocytes with high $(30 \mathrm{mM})$ glucose and a high-affinity ATP-competitive CaMKII inhibitor, AS105 (60). AS105 prevented increased CaMKII activity in response to hyperglycemia. These 
A

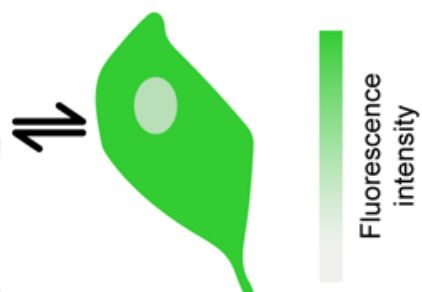

$\frac{\text { Inactive CaMKII }}{\text { † Nuclear import }} \quad \frac{\text { Active CaMKII }}{\uparrow \text { Nuclear export }}$

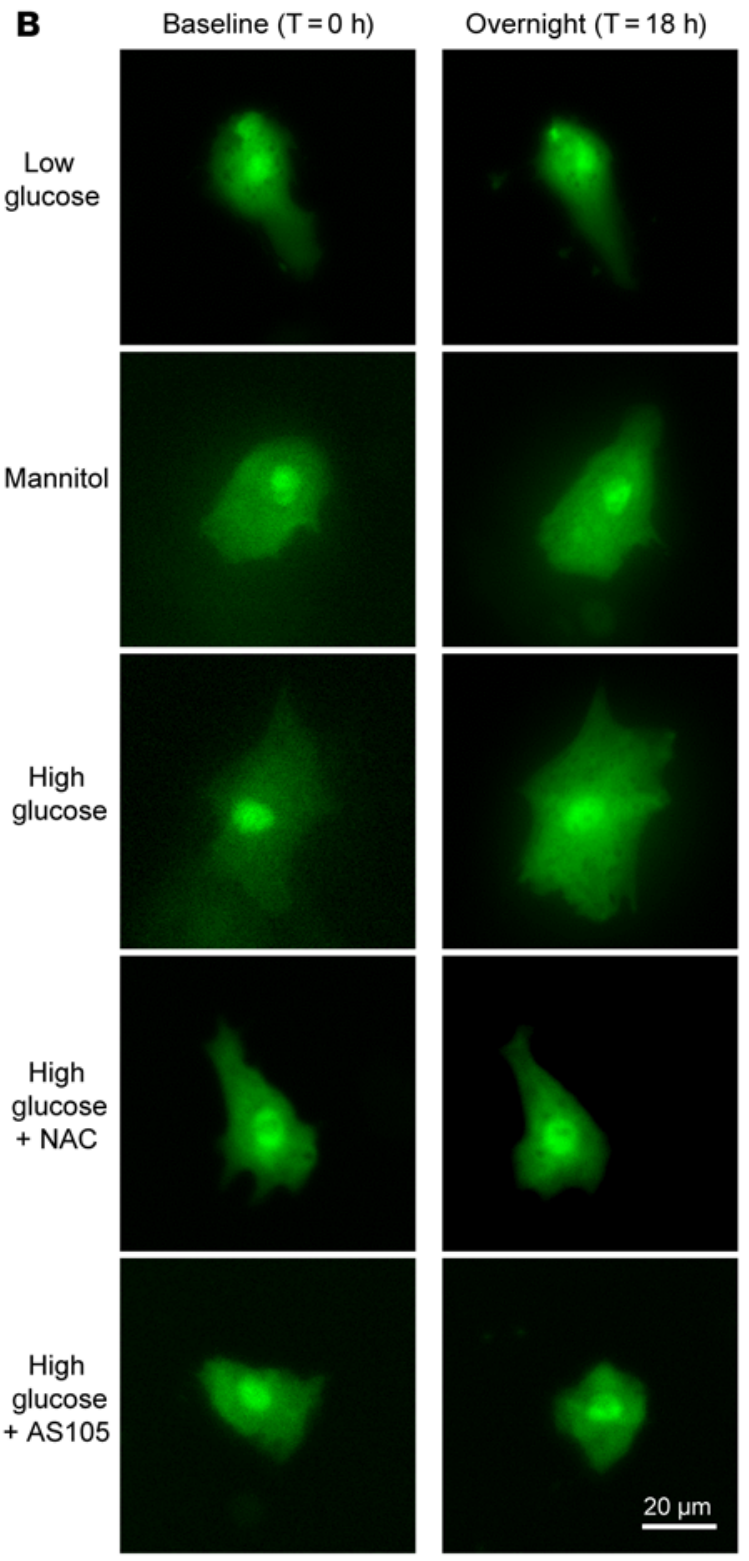

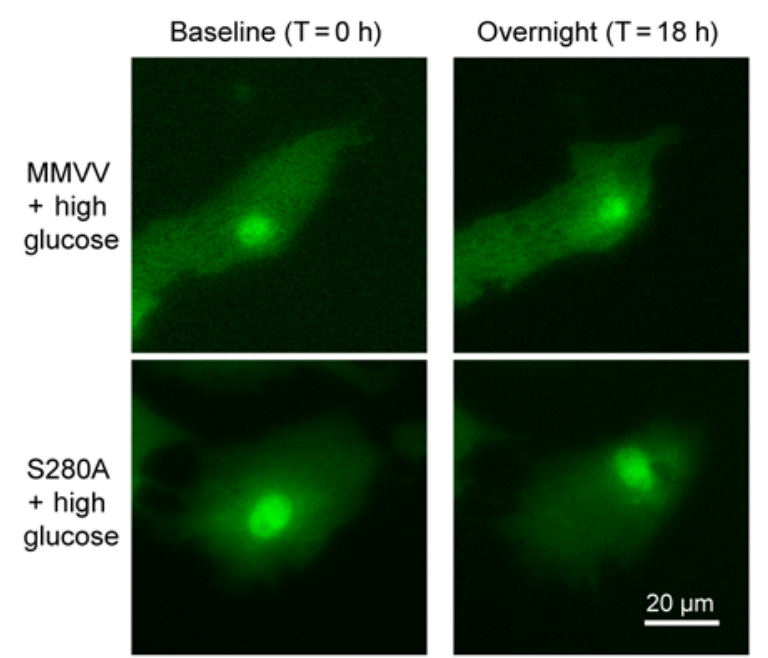
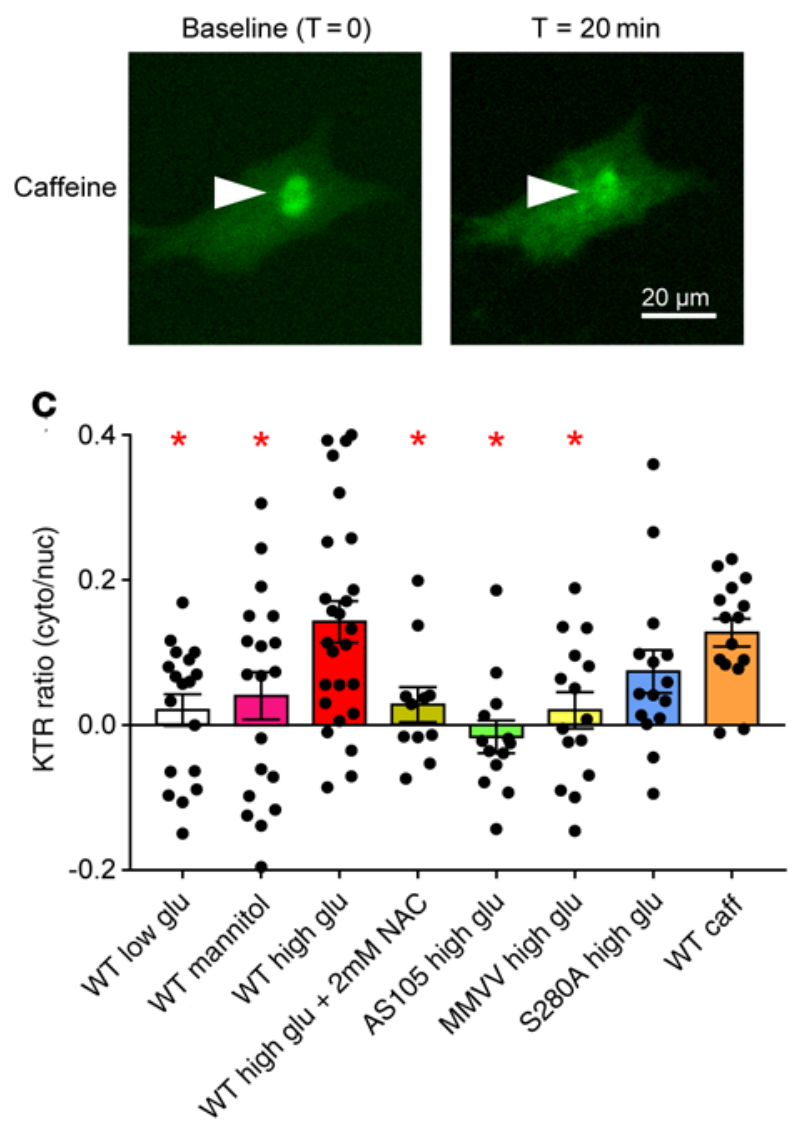
Figure 4. MM281/282 but not S280 is critical for CaMKII activation in response to hyperglycemia. (A) Schematic of CaMKII kinase translocation reporter (CaMKII-KTR) assay. CaMKII-KTR traffics between the nucleus and cytoplasm and phosphorylation by CaMKII results in net translocation of the KTR to the cytosol. Cytosolic to nuclear fluorescent signal ratio is a measure of CaMKII activity. (B) Representative fluorescent micrographs of KTR transfected neonatal mouse cardiomyocytes at baseline and time, $t=$ 18 hours after treatment. Cells from WT pups were incubated as indicated with $5.5 \mathrm{mM}$ glucose (low glucose, $n=18$ cells), $5.5 \mathrm{mM}$ glucose $+24.5 \mathrm{mM}$ mannitol (mannitol, $n=19$ cells), $30 \mathrm{mM}$ glucose (high glucose, $n=26$ cells), high glucose $+2 \mathrm{mM} \mathrm{N}$-acetyl cysteine (high glucose $+\mathrm{NAC}, n=11$ cells), or high glucose $+1 \mu$ M AS105 (a CaMKII inhibitor, $n=13$ cells). Cells from MMVV ( $n=$ 15 cells) and S280A ( $n=15$ cells) pups were incubated with high glucose. Cells from WT pups at baseline and time, $t=20$ minutes after treatment with 10 $\mathrm{mM}$ caffeine ( $n=15$ cells). Arrowheads indicate nuclei. (C) Summary data of the change in KTR cytosolic/nuclear ratio before and after treatments. Data are represented as mean $\pm \mathrm{SEM}$, and statistical comparisons were performed using 1-way ANOVA with Dunnett's multiple-comparison test $\left({ }^{*} P<0.05\right)$.

findings were consistent with the notion that hyperglycemia activates CaMKII. As a further proof of concept, we incubated WT neonatal cardiomyocytes with caffeine $(10 \mathrm{mM})$, a CaMKII activator, for 20 minutes in the absence of hyperglycemia and observed a similar increase in CaMKII activity compared with high glucose (Figure 4, B and C). Next, to assess the contribution of S280 and MM281/282 (Figure 1A) to CaMKII activation by hyperglycemia, we incubated neonatal cardiomyocytes isolated from CaMKII $\delta$ MMVV and S280A pups in high glucose. MMVV-knockin prevented increased CaMKII activation in response to hyperglycemia, whereas the S280A cardiomyocytes exhibited increased CaMKII activity in response to hyperglycemia (Figure 4, B and C). Given the apparent importance of ROS and MM281/282 in activating CaMKII under hyperglycemic conditions, we co-incubated WT neonatal cardiomyocytes with high glucose and $N$-acetyl cysteine (NAC). Treatment with $2 \mathrm{mM}$ NAC prevented CaMKII activation in response to hyperglycemia. Taken together, these findings demonstrated that CaMKII activation by hyperglycemia required MM281/282 (ox-CaMKII) and ROS in neonatal cardiomyocytes.

To further resolve OGN modification at S280 on CaMKII under euglycemic and hyperglycemic conditions, we performed targeted mass spectrometry analysis on CaMKII gel bands and immunoprecipitates from heart lysates from WT nondiabetic, T1D, and T2D mice. Specifically, we focused on the region of interest in the regulatory domain of the CaMKII peptide with the amino acid sequence STVASMMHR (Figure 1, A and B), with several candidate posttranslational modification sites. We reliably detected 2 isoforms of CaMKII, Camk2 $g$ and Camk2d, with good coverage ( $46 \%$ to $58 \%$ ) in the heart samples (Supplemental Table 3). We did not detect OGN modification at S280, despite several attempts using both data-dependent and targeted mass spectrometry methods. In addition, we did not detect OGN modification at other sites on CaMKII. We were, however, able to detect HexNAc sugar modification on oxonium fragment ions on a different protein in these CaMKII-enriched samples (data not shown). Interestingly, we detected phosphorylation at S280 on Camk2g isoform in T1D hearts and on Camk2d (the predominant isoform in the heart) and Camk2g isoforms in T2D hearts (Supplemental Figure 5, A and B, and Supplemental Tables 3 and 4). In confirmation of prior studies on CaMKII posttranslational modification, we identified oxidation at the paired methionines at MM281/282 and phosphorylation at T287, the first identified CaMKII posttranslational modification (61) (Supplemental Figure 5, A-E, and Supplemental Tables 3-5). We detected additional potential phosphorylation sites at S276 or T277 on the Camk2 $g$ isoform in T2D hearts (Supplemental Figure $5 \mathrm{C}$ and Supplemental Tables 3 and 5). Although these findings do not rule out OGN modification at S280, our inability to detect this posttranslational modification is consistent with our findings that S280A mice with T1D or T2D were not protected from AF. Further, these findings suggest that effects of hyperglycemia via OGN modification are independent of CaMKII.

Attenuation of ROS signaling prevents AF due to hyperglycemia. Unlike mutation of S280, manipulation of M281/282 consistently demonstrated that methionine oxidation promoted AF in both diabetic models. We next focused on the T1D model, because of its relative simplicity, to test for cellular sources of ROS that were important for ox-CaMKII- and hyperglycemia-induced AF. Major ROS sources in the heart include mitochondria and NADPH oxidases. We used a combination of genetic and pharmacological approaches to inhibit ROS. Based on our earlier discovery that mitochondrial ROS increases ox-CaMKII in vitro during hyperglycemia (39), we tested whether mitochondrial antioxidant therapy could protect against AF in T1D. We found that mice treated with daily injections of MitoTEMPO (1 mg/kg, i.p.), a mitochondrial targeted antioxidant (62), for 7 days, starting 1 week after STZ injection, were protected from AF in T1D (Figure 5A). In contrast, T1D mice treated with triphenylphosphonium (TPP), the mitochondrial targeting moiety of MitoTEMPO that lacks the antioxidant TEMPO, were not protected from AF (Figure 5A). MitoTEMPO- and TPP-treated mice had similar levels of hyperglycemia (Figure $5 \mathrm{~B})$. We confirmed increased mitochondrial ROS in atrial myocytes isolated from T1D using MitoSOX (Figure 5C), a fluorescent probe used for detection of mitochondrial ROS. NADPH oxidase is another important source of extramitochondrial ROS for increasing ox-CaMKII and promoting $\operatorname{AF}(20,23,63)$. Mice with T1D lacking $\mathrm{p} 47$ ( $p 7^{-/-}$mice), an important component of myocardial NADPH oxidases (64), were also protected from AF (Figure 5A) and had similar levels of hyperglycemia as WT T1D mice (Figure $5 \mathrm{~B})$. Taken together, these data suggested that reduction of ROS signaling either by NADPH oxidase or mitochondrial ROS in the setting of T1D was sufficient to reduce AF risk.

ox-CaMKII can be reduced by methionine sulfoxide reductase A (MsrA), and mice lacking MsrA ( $\mathrm{MrrA}^{-/-}$mice) show increased ox-CaMKII (23). In contrast, mice with transgenic myocardial overexpression of MsrA are protected from ox-CaMKII-induced cardiomyopathy (65). We found that nondiabetic $\mathrm{MsrA}^{-/-}$mice had easily induced AF, similar to WT T1D mice (Figure 5A), and that $M s r A$-transgenic mice with T1D were protected against AF, similar to nondiabetic WT mice (Figure 5A). The antiarrhythmic responses to mitoTEMPO infusion or MsrA overexpression were not related to effects on hyperglycemia because blood glucose levels were similarly increased in all mice (Figure 5B). We interpreted these findings as an indication that methionine oxidation and ox-CaMKII are elements of a conserved proarrhythmic pathway downstream to ROS in T1D. Further, decreasing methionine reductive capacity was sufficient to increase AF in the absence of DM. 
A

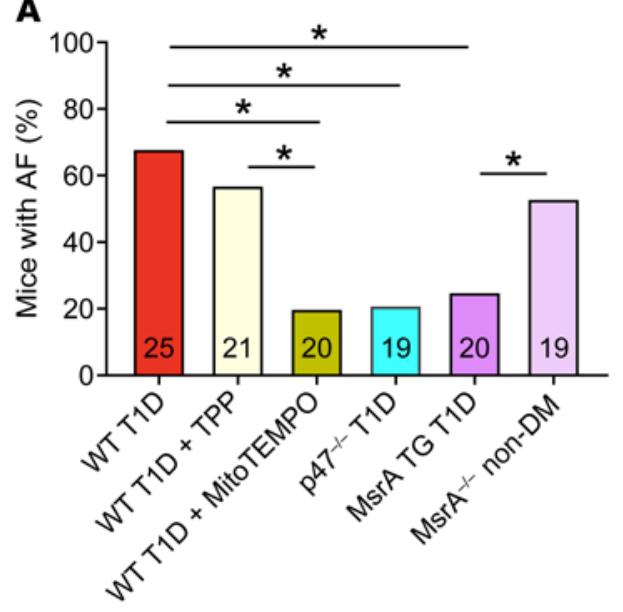

C
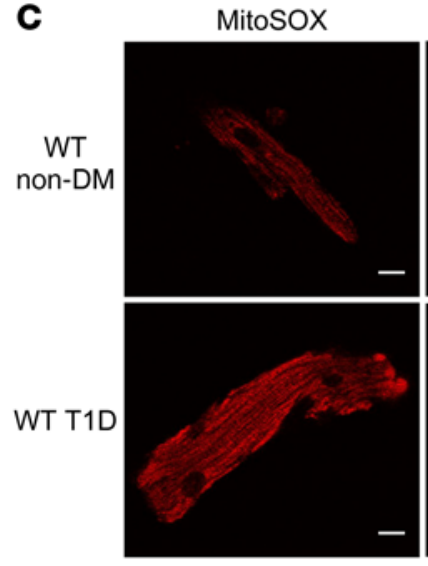

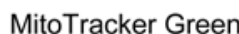
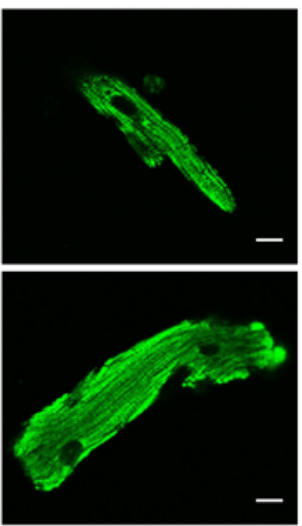

B
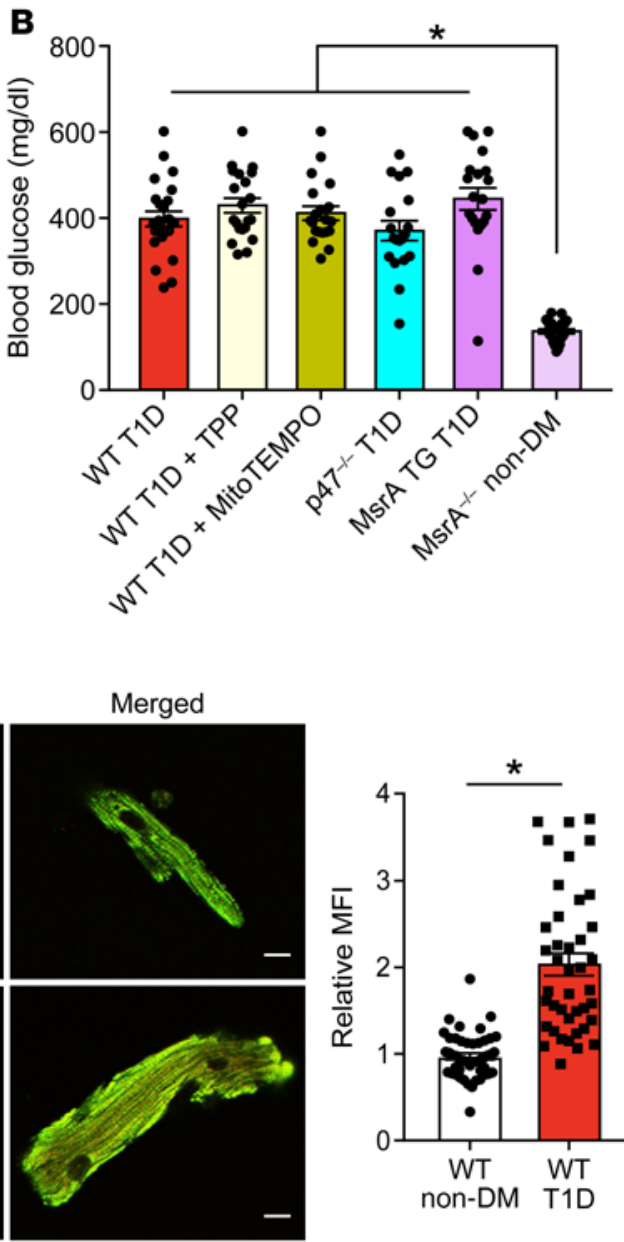

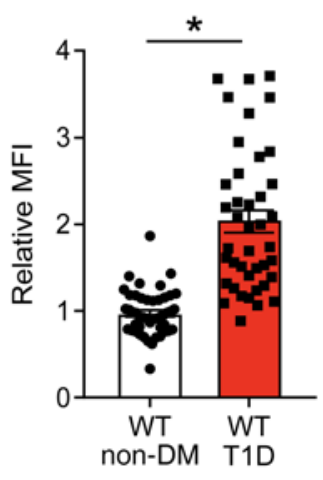

Figure 5. Targeted ROS inhibition and MsrA overexpression protects against atrial fibrillation in diabetes. (A) Inhibition of mitochondrial ROS by MitoTEMPO treatment or inhibition of cytoplasmic ROS by loss of the p47 subunit of NADPH oxidase ( $p 47^{-/-}$mice) protect against AF in T1D mice. Mice with myocardial targeted transgenic overexpression of methionine sulfoxide reductase A (MsrA TC) were protected from T1D primed AF; nondiabetic mice lacking MsrA (MrsA ${ }^{-/-}$ non-DM) showed increased AF susceptibility in the absence of diabetes. (B) Summary data of blood glucose measurements at the time of electrophysiology study. (C) Increased mitochondrial ROS in isolated atrial myocytes from WT T1D (bottom) compared with WT non-DM (top) mice detected by MitoSOX fluorescence. Representative confocal fluorescent images (original magnification, $\times 40$ ) show MitoSOX (red, left), MitoTracker (green, middle), and merged images (right). Scale bars: $10 \mu \mathrm{m}$. ( $n=41-47$ cells in each group from 2 mice per group). Data are represented as percentage frequency distribution (A) and mean \pm SEM (B and $\mathbf{C}$ ). The numerals in the bars represent the sample size in each group (A). Statistical comparisons were performed using 2-tailed Fischer's exact test with Holm-Bonferroni correction for multiple comparisons (A), 1-way ANOVA with Tukey's multiple-comparison test (B) and 2-tailed Student's $t$ test (C). $\left.{ }^{*} P<0.05\right)$. WT T1D data set (A and $\mathbf{B})$, control data previously presented. AF, atrial fibrillation; DM, diabetes mellitus; T1D, type 1 DM; T2D, type 2 DM.

Targeted OGN inhibition is protective against AF susceptibility in T1D and T2D. Our studies up to this point did not address the possibility that increased OGN in T1D and T2D (Figure 2B) is proarrhythmic, potentially by actions at targets other than CaMKIII. As a first step, we asked whether preventing the synthesis of the $O$-GlcNAc transferase substrate UDP-GlcNAc with the glutamine-fructose amidotransferase antagonist 6-diazo-5-oxo-L-norleucine (DON) could reduce or prevent $\mathrm{AF}$ in diabetic mice. We administered DON (5 mg/kg, i.p. injection) 30 minutes prior to $\mathrm{AF}$ induction. DON treatment significantly reduced AF susceptibility in WT T1D mice and S280A T1D mice (Figure 6A). MMVV T1D mice treated with DON had no significant further decrease in AF susceptibility (Figure 6A). These findings suggested that OGN contributed to diabetic AF in the T1D mouse model, but that this was independent of the S280 site on CaMKIIס. We next asked whether OGN inhibition had antiarrhythmic actions in T2D by treating $\mathrm{T} 2 \mathrm{D}$ mice with $\mathrm{DON}$ prior to $\mathrm{AF}$ induction, as in the earlier T1D studies. Surprisingly, DON treatment was not protective against AF in T2D (Figure 6B). We interpreted these data up to this point to highlight important but unanticipated differences in the AF mechanisms in T1D and T2D, at least in mice.

We recognized that the results showing AF suppression by DON needed to be interpreted with caution. Although it is a widely used tool inhibitor, DON has the potential for off-target effects (66). We thus developed a transgenic mouse model with myocardial overexpression of OGA (OGA-TG mice) (Figure 6, C and D), as an orthogonal approach to testing for the therapeutic potential of reducing myocardial OGN in diabetic AF. The OGA-TG mice were born in Mendelian ratios and had normal morphology, and they had similar body weight and blood glucose at baseline compared with WT littermates (Supplemental Figure 6, A and B). These mice had normal echocardiographic features, including LV fractional shortening, 
A

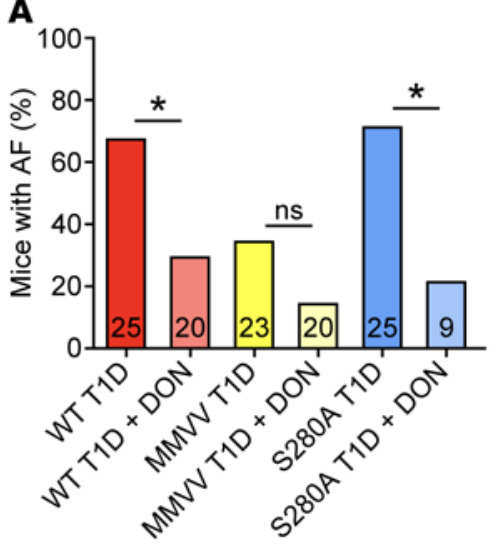

D

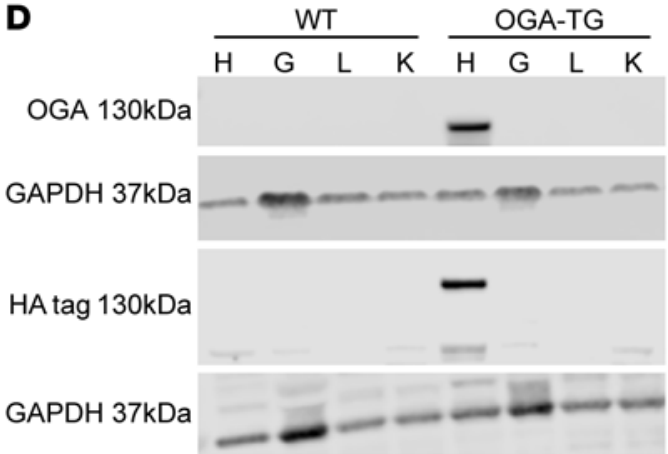

B

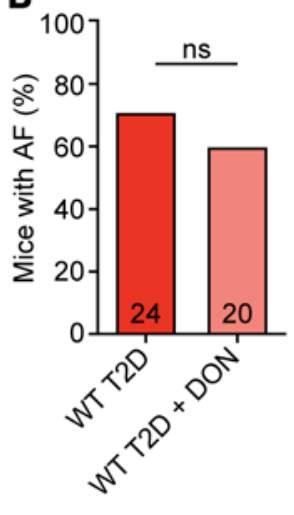

C

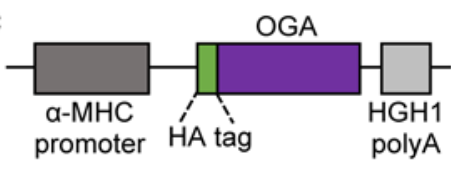

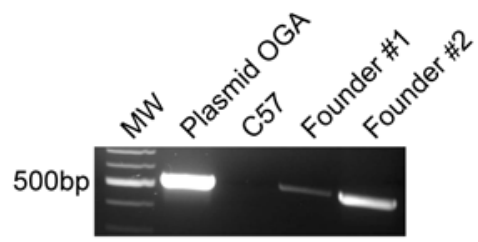

E

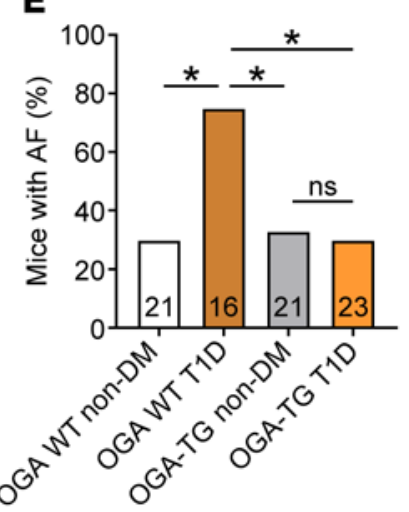

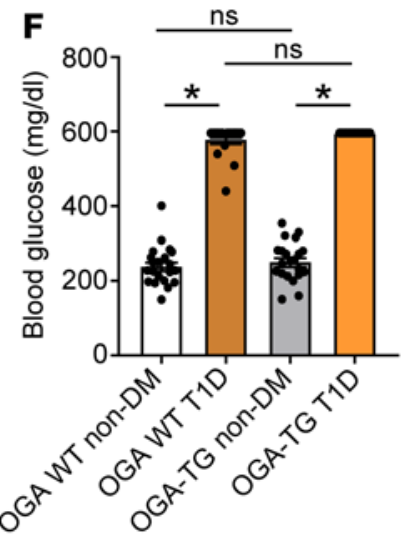

G

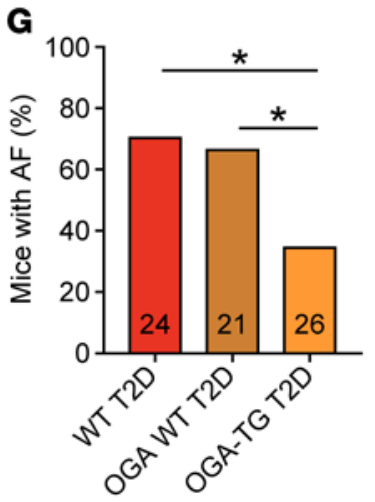

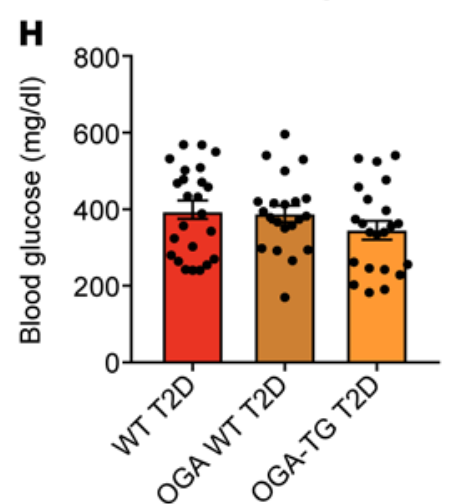

Figure 6. Targeted OGN inhibition is protective against atrial fibrillation susceptibility in type 1 and type 2 diabetes. (A) DON pretreatment (5 mg/kg i.p.) protected from AF in T1D WT and S280A mice, with no additional protection in T1D MMVV mice. (B) DON pretreatment did not protect from AF in T2D WT mice. (C) Schematic of the OGA ( $O$-GIcNAcase) transgene construct with the $\alpha$-myosin heavy chain ( $\alpha$-MHC) promoter, HA epitope marker, and human growth hormone polyA signal ( $\mathrm{HGH1}$ ) (top). PCR product validation of OGA transgene expression in 2 founder pups (OCA-transgenic mice, OGA-TG). The line with the higher OCA expression was chosen for further experiments (bottom). (D) Western blot for OCA transgene and HA epitope expression in heart (H), gastrocnemius muscle (G), liver (L), and kidney (K) from WT and OCA-TG mice. (E) OCA-TC mice were protected from enhanced AF in T1D. (F) OCA-TC mice had similar blood glucose levels as WT littermates under T1D and nondiabetic conditions. (G) OCA-TG were protected from enhanced AF in T2D. (H) OCA-TG mice had similar blood glucose levels as WT T2D mice. DON, 6-diazo-5-oxo-L-norleucine; AF, atrial fibrillation; DM, diabetes mellitus; T1D, type 1 DM; T2D, type 2 DM. Data are represented as percentage frequency distribution ( $\mathbf{A}, \mathbf{B}, \mathbf{E}$, and $\mathbf{G})$ and mean \pm SEM (F and $\mathbf{H})$. The numerals in the bars represent the sample size in each group $(\mathbf{A}$, $\mathbf{B}, \mathbf{E}$, and G). Statistical comparisons were performed using 2-tailed Fischer's exact test with Holm-Bonferroni correction for multiple comparisons (A, B, E, and G), 1-way ANOVA with Tukey's multiple-comparison test (F and $\mathbf{H}) .\left({ }^{*} P<0.05\right)$. WT T1D $(\mathbf{A})$ and T2D (B, G, and $\left.\mathbf{H}\right)$ data sets are control data previously presented.

mass, and dimensions (Supplemental Figure 6, C-H) and similar resting heart rates (Supplemental Figure 6I) and heart weight indexed for body weight (Supplemental Figure 6J) compared with WT littermates. The OGA-TG mice were protected from increased AF in T1D compared with WT T1D mice (Figure 6E) and had similar levels of hyperglycemia as WT T1D mice (Figure 6F). Similarly,
OGA-TG mice were protected from increased AF in T2D compared with WT T2D mice (Figure 6G) with similar levels of hyperglycemia as the WT T2D mice (Figure 6H). We interpreted these data to show that OGN signaling likely contributes to AF in T1D and T2D.

ox-CaMKII enhances triggered activity and $\mathrm{RyR} 2 \mathrm{Ca}^{2+}$ leak in diabetic AF. CaMKII is proarrhythmic, in part, by promoting 
afterdepolarizations $(21,22,67)$. Delayed afterdepolarizations (DADs) are observed as membrane-potential fluctuations that occur after action-potential repolarization (68). To test whether DADs were increased in atrial myocytes from T1D mice, we rapidly paced isolated atrial myocytes to simulate the model of AF induction by rapid atrial pacing using patch clamp in current clamp mode (Supplemental Figure 7A). Atrial myocytes isolated from WT T1D mice exhibited increased frequency of DADs and spontaneous action potentials compared with nondiabetic controls (Supplemental Figure 7B). MMVV T1D mice were resistant to increased DADs and action potentials (Supplemental Figure 7B). We interpret these data to indicate that ox-CaMKII augments DADs in T1D, a potential cellular mechanism explaining increased propensity for AF in DM.

Excessive RyR2 $\mathrm{Ca}^{2+}$ leak has emerged as a core proarrhythmic mechanism that is activated by CaMKII (69). Furthermore, RyR2 is a candidate arrhythmia target downstream to OGN and ox-CaMKII because DON reduced RyR2 $\mathrm{Ca}^{2+}$ leak in ventricular cardiomyocytes exposed to hyperglycemia and suppressed arrhythmias in diabetic rats (24), and MMVV mouse atrial cardiomyocytes were resistant to angiotensin II-triggered RyR2 $\mathrm{Ca}^{2+}$ leak (20). RyR2 S2814 is a validated CaMKII site (69), and CaMKII-catalyzed hyperphosphorylation of S2814 contributes to increased RyR2 $\mathrm{Ca}^{2+}$ leak, DADs, and triggered arrhythmias $(21,22,67)$. Furthermore, S2814A-knockin mice are resistant to angiotensin II-primed AF, a model where ox-CaMKII and RyR2 S2814 are required for proarrhythmia (20). We found increased $\mathrm{Ca}^{2+}$ sparks (Supplemental Figure 7C) in atrial myocytes isolated from WT T1D mice (Supplemental Figure 7, C and D), but not from MMVV T1D mice (Supplemental Figure 7, C and $\mathrm{D}$ ). We found that the S2814A mice were protected from AF in both T1D and T2D (Supplemental Figure 7, E and F). We interpreted these data as consistent with the concept that T1D and T2D augment a CaMKII-RyR2 proarrhythmic signaling pathway.

Although ourdata indicates AF wasincreased in diabetic mice by apathwaythatenhanced cellulartriggering, AFcanalsobesupported by structural tissue-level changes, such as cell death and fibrosis, which favor formation of electrical reentrant circuits $(67,70,71)$. Furthermore, excessive CaMKII activity can contribute to myocardial death and fibrosis $(65,72)$. We performed histological analysis of atrial tissue from WT mice with and without T1D (Supplemental Figure 8, A and B), but did not detect differences in H\&E staining or fibrosis. We measured cleaved caspase 3, a marker of apoptosis (73), and did not detect differences between WT nondiabetic and WT T1D mice (Supplemental Figure 8C). We interpret these data to suggest that differences in pathological atrial remodeling were unlikely to contribute to AF in our model, most likely because of the brief, 2-week duration of T1D in this model.

\section{Discussion}

Our studies provide evidence that ox-CaMKII and OGN play critical but independent roles in the mechanism for increased AF in DM. We found increased AF in T1D and T2D mouse models, and this increased susceptibility to AF was absent in mice with myocardial CaMKII inhibition. Thus, CaMKII appears to be a node connecting upstream signals in T1D and T2D with downstream arrhythmia mechanisms. We confirmed previous observations that ROS activates CaMKII, but in contrast to prior studies, our data did not support a role for $O$-GlcNAc in activation of CaMKII. Notably, loss of CaMKII $\delta$ S280, the previously identified OGN site for CaMKII (24), was ineffective in preventing AF in T1D and T2D. To our knowledge, there are no in vivo data showing CaMKII modification by OGN at S280 or other sites. We were unable to detect this modification on CaMKII from heart lysates isolated from mice with or without T1D and T2D, but we did detect phosphorylation at S280 in Camk2g and Camk2d. At this point, it is uncertain whether S280 phosphorylation can confer $\mathrm{Ca}^{2+}$ - and calmodulin-autonomous activity on CaMKII, so future studies will be necessary to test for this possibility.

We demonstrated that OGN contributed to AF in T1D and T2D. DON, a competitive inhibitor of the hexosamine biosynthetic pathway that reduces availability of UDP-GlcNAc, the rate-limiting substrate for OGN, was effective at suppressing $\mathrm{AF}$ in T1D but not T2D, suggesting that OGN was proarrhythmic in T1D by targeting sites other than CaMKII, and/or that off-target actions of DON were antiarrhythmic. In contrast to our observations in DON-treated mice, our finding that OGA-TG mice were protected from $\mathrm{AF}$ in both $\mathrm{T} 1 \mathrm{D}$ and $\mathrm{T} 2 \mathrm{D}$ suggests that $\mathrm{DON}$ is antiarrhythmic, at least in part by off-target actions that are independent of CaMKII and OGN. We interpret the shared resistance to AF by AC3-I, MMVV, and OGA-TG mice in T1D and T2D to suggest that CaMKII $\delta$ and OGN are proarrhythmic in DM.

The response to loss of ox-CaMKII $\delta$ was remarkably consistent. The increased susceptibility to AF was similarly suppressed in T1D and T2D in MMVV mice. Upstream of CaMKIIS, NADPH oxidase and mitochondrial ROS are major sources of intracellular ROS in physiology and disease (74). In this study, we observed protection from $\mathrm{AF}$ in T1D in $p 47^{-/-}$mice deficient in NADPH oxidase, similar to our previous finding that preventing oxidative activation of CaMKII $\delta$ downstream of NADPH oxidase was antiarrhythmic in an angiotensin II-primed model of AF (20). Further, targeted inhibition of mitochondrial ROS with MitoTEMPO also protected against AF in T1D. Inhibition of mitochondrial ROS by genetic overexpression of the human catalase gene targeted to the mitochondria has been shown to decrease AF in a mouse model (75). The observed rescue with either inhibition of NADPH oxidase or mitochondrial ROS is an interesting finding, and is consistent with the idea of crosstalk between intracellular and mitochondrial ROS production in the feed-forward mechanism known as ROS-induced ROS production (76). Although our study is focused on the role of CaMKII, we acknowledge that there are other intracellular targets of oxidation that may play a role in the mechanism of oxidative stress-related AF. Indeed, Xie et al. demonstrated that RyR2 oxidation contributes to the pathogenesis of $\mathrm{AF}$ (75). We interpret these findings to support an important role for CaMKII as a transducer of pathological ROS in AF in the setting of DM. Although we only tested the role of candidate ROS sources and MsrA in T1D, it seems plausible that future studies will confirm that targeted antioxidant interventions upstream to CaMKII have the potential to reduce AF risk in T1D and T2D.

At present, there are no CaMKII-based therapies available for clinical use, but there is evidence that OGN-based therapeutics are advancing to the clinical setting (77-79). The potential that such agents may soon become available is exciting, in part because they may have broad application, including as antiarrhythmic drugs for $\mathrm{AF}$ in patients with DM. If our findings that OGA-TG mice were resistant to $\mathrm{AF}$ in $\mathrm{DM}$ have translational implications for humans, 
then a possible advantage of OGN-based therapies is that they may be successful in T1D and T2D, likely affecting a diverse array of protein targets. OGN targets myriad proteins and pathways implicated in myocardial disease, such as transcription factors (33), myofilament proteins $(36,80)$, and mitochondrial metabolism $(81)$. OGN is known to participate in physiological processes (33) and is an essential stress response. Mice lacking OGT show augmented pathological responses to cardiac injury (82). Thus, the breadth of OGN substrates and the requirement for increased OGN to overcome myocardial injury should strike a cautionary note concerning the potential for unintended off- and on-target actions with OGN-based therapeutics. Availability of drugs or tool molecules with drug-like properties will constitute an important step in determining when, how, and if OGN therapeutics will be applicable to patients with diabetes at risk for AF.

Clinically, the sodium-glucose cotransporter 2 (SGLT2) inhibitors, such as empagliflozin and dapagliflozin, which were developed primarily as glucose-lowering agents in the treatment of T2D, have shown impressive cardiovascular benefits, including mortality and heart failure benefits $(83,84)$. The DAPA-HF clinical trial showed that these benefits are independent of the presence or absence of diabetes (84). This has led to increased interest in the mechanisms underlying these observed beneficial cardiovascular effects, especially because SGLT2 is not expressed in the heart $(85,86)$. A recent subgroup analysis of the DECLARE-TIMI 58 clinical trial showed that dapagliflozin decreased the incidence of AF in high-risk patients with T2D, independent of HbA1c levels (87). In rodent models, dapagliflozin use in diabetic mice resulted in reduction in myocardial OGN levels in treated compared with untreated mice (88), and empagliflozin attenuated atrial remodeling and improved mitochondrial function in a rat model of T2D (89). Taken together, these findings highlight a potential mechanism and therapeutic target focused on OGN by which SGLT2 inhibitors can be a tool for the management of AF independent of the presence or absence of diabetes. Exciting early work by Mustroph et al. demonstrated that empagliflozin reduces CaMKII activity and CaMKII-dependent SR $\mathrm{Ca}^{2+}$ leak in isolated ventricular myocytes from a mouse heart failure model and human failing ventricular myocytes (86). However, the mechanisms by which SGLT2 acts through or on CaMKII are unknown, and further work to investigate these mechanisms and their potential antiarrhythmic effects are needed (90).

Our results demonstrated a previously unexplored role for ox-CaMKII and OGN in promoting AF in T1D and T2D, as evidenced by the antiarrhythmic response to loss of CaMKII $\delta$ M281/282 in MMVV-knockin mice or myocardial overexpression of OGA in OGA-TG mice. An unanticipated finding in our study was the lack of antiarrhythmic response with loss of CaMKII $\delta 280$ in S280A-knockin mice and lack of detection of OGN modifications to CaMKII, specifically in the CaMKII regulatory domain, as previously described (24). To our knowledge, this is the first study to assess OGN-CaMKII in in vivo heart samples. Because ROS and OGN are elevated in T1D and T2D, it is possible that CaMKII oxidation and OGN occur together as a hybrid, activating posttranslational modification. However, our findings make this model less probable given the lack of antiarrhythmic response in diabetic S280A mice, but the shared antiarrhythmic response in T1D and T2D MMVV mice. In part because we did not detect OGN at CaMKIIS S280, we currently lack proteomic information to rule in or rule out the possibility that loss of M281/282 impairs S280 OGN or, reciprocally, that loss of S280 affects the redox status of M281/282. However, these scenarios would appear more plausible if there were conserved protection or concordant lack of protection against AF in MMVV and S280A mice with T1D or T2D. Rather, we interpret our findings to indicate separate and independent roles for ox-CaMKII and OGN in hyperglycemia-induced AF and speculate that RyR2 is a potential downstream OGN target. Although important open questions remain, our data identified CaMKII as a unifying cause of AF in DM. These findings, taken together with emergent evidence that CaMKII contributes to multiple aspects of $\mathrm{DM}$, including gluconeogenesis in the liver and insulin signaling in skeletal muscle (91-93), suggest that further study of the role of CaMKII in metabolism and metabolic diseases will be important for improved understanding and treatments.

\section{Methods}

Additional details are provided in Supplemental Methods.

Mouse models. Experimental studies were performed on male mice on a C57BL/6J background. C57BL/6J mice and mice lacking a functional NADPH oxidase $\left(p 47^{--}\right)$were purchased from The Jackson Laboratory. Our lab previously described the generation of AC3-I (50) and MsrA-transgenic (65) mice. We also previously generated MMVV-knockin mice as previously described (39). S2814A-knockin mice were generated in-house $(21,94)$. CaMKII $\delta$-S280A-knockin mice harboring a point mutation in the mouse CaMKII $\delta$ gene to substitute serine 280 with alanine (S280A) were generated on a C57BL/6J background using the CRISPR/Cas9 technology. A single-guide RNA (target sequence: 5'-CTGTTGCCTCCATGATGCACAGG-3') was designed to target CaMKIII. Synthetic ssDNA for CRISPR-homology repair was designed to harbor mutations including S280A (TCC $\rightarrow$ GCG) and NsiI recognition site (ATGCAT). Genotyping of founder mice and generations of offspring was performed initially by both direct sequencing of PCR amplified fragments and PCR genotyping from tail DNA with the following primers: forward, 5'-AGGAAATGCTTGCCAAAGTAGTG-3'; reverse, 5'-CCAGCACATACTGCCCTAGC-3'. Founder S280A mice were backcrossed with C57BL/6J mice from The Jackson Laboratory.

Transgenic mice with myocardial targeted overexpression of OGA (Tg OGA mice) were generated on a C57Bl/6J background. HA-tagged human meningioma expressed antigen 5 (OGlcNAcase, OGA) cDNA was generated in-house and was subcloned into the pBS- $\alpha$ MHC-scripthGH vector between the murine $\alpha \mathrm{MHC}$ promoter and a human growth hormone polyadeneylation sequences. Purified pronuclear injections of linearized DNA (digested with NotI) were performed in the Johns Hopkins Transgenic Mouse Core Facility and embryos implanted into pseudopregnant females to generate C57BL/6J F1 mice. The F1 pups were screened for insertion of the transgene into the mouse genome by PCR analysis (Figure 6C) using the forward primer $5^{\prime}$-TGGTCAGGATCTCTAGATTGGT-3' and reverse primer 5'-TCATAAGTTGCTCAGCTTCCTC- $3^{\prime}$, producing a product of 850 base pairs.

CaMKII KTR assay. Construction and validation of the CaMKII kinase activity reporter (CaMKII-KTR) was recently described by our lab in RPE-1 cells and skeletal muscle fibers (59), based on the initial work by and collaboration with Regot et al. (58). CaMKII-KTR DNA plasmid was transfected (JetPrime Transfection Reagent, PolyPlus) into neonatal mouse cardiomyocytes (isolated from C57BL/6J WT, MMVV, and S280A pups) 24 hours after isolation. After an 18-hour 
transfection incubation, cells were imaged in M199 cell culture medium with 2\% FBS (Thermo Fisher Scientific, 12340030) supplemented for 18 hours through the experiment with low glucose $(5.5 \mathrm{mM})$, high glucose $(33 \mathrm{mM})$, mannitol $(22 \mathrm{mM})$, NAC $(2 \mu \mathrm{M})$, or AS105 $(1 \mu \mathrm{M})$. Caffeine $(10 \mathrm{mM})$ was applied acutely over 20 minutes as a positive control. Fluorescent images were collected using an Olympus IX83 microscope equipped with an ORCA Flash 4.0 sCMOS camera and 2OX UPLFLN2OX NA0.5 objective lens. Cells were maintained at $37^{\circ} \mathrm{C}$ in an OkoLabs stage top incubator. Image analyses were carried out in Image (NIH). The cytosolic to nuclear KTR signal ratios were calculated using the mean intensities measured from the nuclei and cytosolic ring (area immediately perinuclear) of individual cells $(58,59)$.

Statistics. All results are expressed as mean \pm SEM for continuous variables or percentage frequency distribution for dichotomous variables. For continuous variables, statistical analysis was performed using an unpaired Student's $t$ test (2-tailed for 2 groups) or 1-way ANOVA followed by Tukey's or Dunnett's post hoc multiple comparisons (for $\geq 3$ groups). For dichotomous variables, statistical analyses were performed using Fischer's exact test (2-tailed for 2 groups) with Holm-Bonferroni correction for post hoc multiple comparisons (for $\geq 3$ groups). A $P$ value of less than 0.05 was considered statistically significant.

Study approval. The Johns Hopkins University animal care and use committee approved all animal experimental protocols. For human samples, the local IRB of the Goettingen University approved all human procedures and each patient gave written informed consent.

\section{Author contributions}

MEA, OOM, and AGR conceived the project; GWH, PSB, RSA, NEZ, PU, and EDL contributed important input for planning the studies; and MEA and OOM wrote the manuscript. NA and JLP developed the S280A mouse. OOM and AT developed the OGA-TG mouse. XHW provided the S2814A mice. BC, LSS, and YW performed cellular electrophysiological and $\mathrm{Ca}^{2+}$ measurement studies. OOM performed the AF studies. LSM and AGR provided human specimens. OOM, JMG, QW, KRM, EDL, NEZ, and PSB performed CaMKII assays and OGN studies. OOM, EDL, TNB, and RNC performed mass spectrometry studies. OOM and QW performed quantitative PCR studies. All authors edited and approved the final manuscript.

\section{Acknowledgments}

We are grateful to Jinying Yang for her assistance in maintaining mouse colonies and Gianna Bortoli for her assistance with neonatal cell isolation. We are also grateful to Djahida Bedja for performing the mouse echocardiograms. We thank Chip Hawkins and the Johns Hopkins University School of Medicine Transgenic Core Laboratory for their technical expertise in generating transgenic mice. We thank the Johns Hopkins University School of Medicine Mass Spectrometry and Proteomics Facility for technical assistance in the mass spectrometry studies. We also thank Teresa Ruggle, who produced the artwork. We are grateful to Howard Schulman for the gift of the CaMKII inhibitor AS105. This work was funded in part by NIH grants R35 HL140034 to MEA; R01-HL089598, R01-HL091947, and R01-HL117641 to XHW; T32-HL007227 to OOM and PU; and 5K12HL141952-03 to PU, in addition to the Foundation Leducq Career Development Award to AGR. This work was also supported by an American Heart Association (AHA) collaborative science award (17CSA33610107 to MEA and GWH) and an AHA grant (13EIA14560061 to XHW). LSM was supported by the Deutsche Forschungsgemeinschaft Ma 1982/5-1. A Synergy Award from Johns Hopkins University to MEA and GWH and the Johns Hopkins Medicine Discovery Fund also made this work possible.

Address correspondence to: Mark E. Anderson, Department of Medicine, The Johns Hopkins School of Medicine, 1830 E. Monument Street, Suite 9026, Baltimore, Maryland 21287, USA. Phone: 410.955.6642; Email: mark.anderson@jhmi.edu. Or to: Olurotimi O. Mesubi, Division of Cardiology, Department of Medicine, The Johns Hopkins School of Medicine, 600 N. Wolfe Street, Carnegie 592A, Baltimore, Maryland 2128, USA7. Phone: 443.287.8388; Email: olurotimi.mesubi@jhmi.edu.
1. Chugh SS, et al. Worldwide epidemiology of atrial fibrillation: a global burden of disease 2010 study. Circulation. 2014;129(8):837-847.

2. King H, Aubert RE, Herman WH. Global burden of diabetes, 1995-2025: prevalence, numerical estimates, and projections. Diabetes Care. 1998;21(9):1414-1431.

3. Centers for Disease Control and Prevention. National Diabetes Statistics Report: Estimates of Diabetes and Its Burden in the United States, 2014. US Department of Health and Human Services; 2014. https://stacks.cdc.gov/view/cdc/23442/ cdc_23442_DS1.pdf. Accessed November 4, 2020.

4. Miyasaka Y, et al. Secular trends in incidence of atrial fibrillation in Olmsted County, Minnesota, 1980 to 2000, and implications on the projections for future prevalence. Circulation. 2006;114(2):119-125.

5. Naccarelli GV, Varker H, Lin J, Schulman KL. Increasing prevalence of atrial fibrillation and flutter in the United States. Am J Cardiol. 2009;104(11):1534-1539.

6. NCD Risk Factor Collaboration (NCD-RisC). Worldwide trends in diabetes since 1980: a pooled analysis of 751 population-based studies with 4.4 million participants. Lancet. 2016;387(10027):1513-1530.

7. Wild S, Roglic G, Green A, Sicree R, King H. Global prevalence of diabetes: estimates for the year 2000 and projections for 2030. Diabetes Care. 2004;27(5):1047-1053.

8. Fuster V, et al. ACC/AHA/ESC 2006 guidelines for the management of patients with atrial fibrillation: a report of the American College of Cardiology/ American Heart Association task force on practice guidelines and the European Society of Cardiology committee for practice guidelines (writing committee to revise the 2001 guidelines for the management of patients with atrial fibrillation): developed in collaboration with the European Heart Rhythm Association and the Heart Rhythm Society. Circulation. 2006;114(7):e257-e354.

9. Go AS, et al. Prevalence of diagnosed atrial fibrillation in adults: national implications for rhythm management and stroke prevention: the anticoagulation and risk factors in atrial fibrillation (ATRIA) study. JAMA. 2001;285(18):2370-2375. 10. Benjamin EJ, Wolf PA, D’Agostino RB, Silbershatz
H, Kannel WB, Levy D. Impact of atrial fibrillation on the risk of death: the Framingham Heart Study. Circulation. 1998;98(10):946-952.

11. Odutayo A, Wong CX, Hsiao AJ, Hopewell S, Altman DG, Emdin CA. Atrial fibrillation and risks of cardiovascular disease, renal disease, and death: systematic review and meta-analysis. $B M J$. 2016;354:i4482.

12. Dahlqvist S, et al. Risk of atrial fibrillation in people with type 1 diabetes compared with matched controls from the general population: a prospective case-control study. Lancet Diabetes Endocrinol. 2017;5(10):799-807.

13. Kannel WB, Abbott RD, Savage DD, McNamara PM. Epidemiologic features of chronic atrial fibrillation: the Framingham study. N Engl JMed. 1982;306(17):1018-1022.

14. Benjamin EJ, Levy D, Vaziri SM, D’Agostino RB, Belanger AJ, Wolf PA. Independent risk factors for atrial fibrillation in a population-based cohort. The Framingham Heart Study. JAMA. 1994;271(11):840-844.

15. Aksnes TA, Schmieder RE, Kjeldsen SE, Ghani S, Hua TA, Julius S. Impact of new-onset diabetes 
mellitus on development of atrial fibrillation and heart failure in high-risk hypertension (from the VALUE trial). Am J Cardiol. 2008;101(5):634-638.

16. DeVore AD, et al. Hospitalizations in patients with atrial fibrillation: an analysis from ROCKET AF. Europace. 2016;18(8):1135-1142.

17. Du X, et al. Risks of cardiovascular events and effects of routine blood pressure lowering among patients with type 2 diabetes and atrial fibrillation: results of the ADVANCE study. Eur Heart J. 2009;30(9):1128-1135.

18. Camm AJ, et al. 2012 focused update of the ESC guidelines for the management of atrial fibrillation: an update of the 2010 ESC guidelines for the management of atrial fibrillation. Developed with the special contribution of the European Heart Rhythm Association. Eur Heart J. 2012;33(21):2719-2747.

19. Morillo CA, et al. Radiofrequency ablation vs antiarrhythmic drugs as first-line treatment of paroxysmal atrial fibrillation (RAAFT-2): a randomized trial. JAMA. 2014;311(7):692-700.

20. Purohit A, et al. Oxidized $\mathrm{Ca}(2+) /$ calmodulindependent protein kinase II triggers atrial fibrillation. Circulation. 2013;128(16):1748-1757.

21. Chelu MG, et al. Calmodulin kinase II-mediated sarcoplasmic reticulum $\mathrm{Ca} 2+$ leak promotes atrial fibrillation in mice. J Clin Invest. 2009;119(7):1940-1951.

22. Neef S, et al. CaMKII-dependent diastolic SR $\mathrm{Ca} 2+$ leak and elevated diastolic $\mathrm{Ca} 2+$ levels in right atrial myocardium of patients with atrial fibrillation. Circ Res. 2010;106(6):1134-1144.

23. Erickson JR, et al. A dynamic pathway for calcium-independent activation of CaMKII by methionine oxidation. Cell. 2008;133(3):462-474

24. Erickson JR, et al. Diabetic hyperglycaemia activates CaMKII and arrhythmias by O-linked glycosylation. Nature. 2013;502(7471):372-376

25. Wu Y, Roden DM, Anderson ME. Calmodulin kinase inhibition prevents development of the arrhythmogenic transient inward current. Circ Res. 1999;84(8):906-912.

26. Sommese L, et al. Ryanodine receptor phosphorylation by CaMKII promotes spontaneous $\mathrm{Ca}(2+)$ release events in a rodent model of early stage diabetes: the arrhythmogenic substrate. Int $J$ Cardiol. 2016;202:394-406.

27. Wagner S, et al. Reactive oxygen species-activated $\mathrm{Ca} /$ calmodulin kinase II $\delta$ is required for late $\mathrm{I}(\mathrm{Na})$ augmentation leading to cellular $\mathrm{Na}$ and $\mathrm{Ca}$ overload. Circ Res. 2011;108(5):555-565.

28. Lu S, et al. Hyperglycemia acutely increases cytosolic reactive oxygen species via O-linked GlcNAcylation CaMKII activation in mouse ventricular myocytes. Circ Res. 2020;126(10):e80-e96.

29. Shah MS, Brownlee M. Molecular and cellular mechanisms of cardiovascular disorders in diabetes. Circ Res. 2016;118(11):1808-1829.

30. Faria A, Persaud SJ. Cardiac oxidative stress in diabetes: mechanisms and therapeutic potential. Pharmacol Ther. 2017;172:50-62.

31. Dassanayaka S, Jones SP. O-GlcNAc and the cardiovascular system. Pharmacol Ther 2014;142(1):62-71.

32. Hart GW, Housley MP, Slawson C. Cycling of O-linked beta-N-acetylglucosamine on nucleocytoplasmic proteins. Nature.
2007;446(7139):1017-1022.

33. Hart GW, Slawson C, Ramirez-Correa G, Lagerlof O. Cross talk between O-GlcNAcylation and phosphorylation: roles in signaling, transcription, and chronic disease. Annu Rev Biochem. 2011;80:825-858.

34. Anderson EJ, Kypson AP, Rodriguez E, Anderson CA, Lehr EJ, Neufer PD. Substrate-specific derangements in mitochondrial metabolism and redox balance in the atrium of the type 2 diabetic human heart. JAm Coll Cardiol. 2009;54(20):1891-1898.

35. Jensen RV, Zachara NE, Nielsen PH, Kimose HH Kristiansen SB, Bøtker HE. Impact of O-GlcNAc on cardioprotection by remote ischaemic preconditioning in non-diabetic and diabetic patients. Cardiovasc Res. 2013;97(2):369-378

36. Ramirez-Correa GA, et al. Removal of abnormal myofilament $\mathrm{O}-\mathrm{GlcNA}$ cylation restores $\mathrm{Ca} 2+$ sensitivity in diabetic cardiac muscle. Diabetes. 2015;64(10):3573-3587.

37. Kronlage M, et al. O-GlcNAcylation of histone deacetylase 4 protects the diabetic heart from failure. Circulation. 2019;140(7):580-594.

38. Yoo $\mathrm{S}$, et al. Oxidative stress creates a unique, CaMKII-mediated substrate for atrial fibrillation in heart failure. JCI Insight. 2018;3(21):e120728.

39. Luo M, et al. Diabetes increases mortality after myocardial infarction by oxidizing CaMKII. JClin Invest. 2013;123(3):1262-1274.

40. Hasslacher C, Wahl P. Diabetes prevalence in patients with bradycardiac arrhythmias. Acto Diabetol Lat. 1977;14(5-6):229-234.

41. Fang ZY, Prins JB, Marwick TH. Diabetic cardiomyopathy: evidence, mechanisms, and therapeutic implications. Endocr Rev. 2004;25(4):543-567.

42. Hsueh W, et al. Recipes for creating animal models of diabetic cardiovascular disease. Circ Res. 2007;100(10):1415-1427.

43. Bullard KM, et al. Prevalence of diagnosed diabetes in adults by diabetes type - United States, 2016. MMWR Morb Mortal Wkly Rep. 2018;67(12):359-361.

44. Lillioja $\mathrm{S}$, et al. Insulin resistance and insulin secretory dysfunction as precursors of noninsulin-dependent diabetes mellitus. Prospective studies of Pima Indians. N EnglJMed. 1993;329(27):1988-1992.

45. Matthews DR, Hosker JP, Rudenski AS, Naylor BA, Treacher DF, Turner RC. Homeostasis model assessment: insulin resistance and betacell function from fasting plasma glucose and insulin concentrations in man. Diabetologia. 1985;28(7):412-419.

46. Wakili R, et al. Multiple potential molecular contributors to atrial hypocontractility caused by atrial tachycardia remodeling in dogs. Circ Arrhythm Electrophysiol. 2010;3(5):530-541.

47. Fischer TH, et al. Late INa increases diastolic SR-Ca2+-leak in atrial myocardium by activating PKA and CaMKII. Cardiovasc Res. 2015;107(1):184-196.

48. Verheule S, et al. Increased vulnerability to atrial fibrillation in transgenic mice with selective atria fibrosis caused by overexpression of TGF-beta1. Circ Res. 2004;94(11):1458-1465.

49. Yi F, et al. Down-regulation of the small conductance calcium-activated potassium channels in diabetic mouse atria. J Biol Chem. 2015;290(11):7016-7026.

50. Zhang R, et al. Calmodulin kinase II inhibition protects against structural heart disease. $\mathrm{Nat}$ Med.2005;11(4):409-417.

51. Anderson ME, Brown JH, Bers DM. CaMKII in myocardial hypertrophy and heart failure. J Mol Cell Cardiol. 2011;51(4):468-473.

52. Swaminathan PD, Purohit A, Hund TJ, Anderson ME. Calmodulin-dependent protein kinase II: linking heart failure and arrhythmias. Circ Res. 2012;110(12):1661-1677.

53. Hoch B, Meyer R, Hetzer R, Krause EG, Karczewski P. Identification and expression of delta-isoforms of the multifunctional $\mathrm{Ca} 2+/$ calmodulin-dependent protein kinase in failing and nonfailing human myocardium. Circ Res. 1999;84(6):713-721.

54. Colomer JM, Mao L, Rockman HA, Means AR. Pressure overload selectively up-regulates $\mathrm{Ca} 2+$ / calmodulin-dependent protein kinase II in vivo. Mol Endocrinol. 2003;17(2):183-192.

55. Wang $\mathrm{H}$, et al. One-step generation of mice carrying mutations in multiple genes by CRISPR/Cas-mediated genome engineering. Cell. 2013;153(4):910-918

56. Hamblet CE, Makowski SL, Tritapoe JM, Pomerantz JL. NK Cell maturation and cytotoxicity are controlled by the intramembrane aspartyl protease SPPL3. JImmunol. 2016;196(6):2614-2626.

57. Yang H, Wang H, Jaenisch R. Generating genetically modified mice using CRISPR/ Cas-mediated genome engineering. Nat Protoc. 2014;9(8):1956-1968.

58. Regot S, Hughey JJ, Bajar BT, Carrasco S, Covert MW. High-sensitivity measurements of multiple kinase activities in live single cells. Cell. 2014;157(7):1724-1734.

59. Wang $\mathrm{Q}$, et al. CaMKII oxidation is a performance/disease trade-off in vertebrate evolution [preprint]. doi: https://doi.org/10.1101/767525. Posted on bioRxiv September 13, 2019.

60. Neef S, et al. Improvement of cardiomyocyte function by a novel pyrimidine-based CaMKII-inhibitor. J Mol Cell Cardiol. 2018;115:73-81.

61. Lou LL, Schulman H. Distinct autophosphorylation sites sequentially produce autonomy and inhibition of the multifunctional $\mathrm{Ca} 2+/$ calmodulin-dependent protein kinase. J Neurosci. 1989;9(6):2020-2032.

62. Murphy MP, Smith RA. Targeting antioxidants to mitochondria by conjugation to lipophilic cations. Annu Rev Pharmacol Toxicol. 2007;47:629-656.

63. Reilly SN, et al. Atrial sources of reactive oxygen species vary with the duration and substrate of atrial fibrillation: implications for the antiarrhythmic effect of statins. Circulation. 2011;124(10):1107-1117.

64. Jackson SH, Gallin JI, Holland SM. The p47phox mouse knock-out model of chronic granulomatous disease. J Exp Med. 1995;182(3):751-758.

65. He BJ, et al. Oxidation of CaMKII determines the cardiotoxic effects of aldosterone. Nat Med. 2011;17(12):1610-1618.

66. Lemberg KM, Vornov JJ, Rais R, Slusher BS. We're not "DON" yet: optimal dosing and prodrug delivery of 6-diazo-5-oxo-L-norleucine. Mol Cancer Ther. 2018;17(9):1824-1832. 
67. Li N, et al. Inhibition of CaMKII phosphorylation of RyR2 prevents induction of atrial fibrillation in FKBP12.6 knockout mice. Circ Res. 2012;110(3):465-470.

68. Schlotthauer K, Bers DM. Sarcoplasmic reticulum $\mathrm{Ca}(2+)$ release causes myocyte depolarization. Underlying mechanism and threshold for triggered action potentials. Circ Res. 2000;87(9):774-780.

69. Wehrens XH, Lehnart SE, Reiken SR, Marks AR. $\mathrm{Ca} 2+/$ calmodulin-dependent protein kinase II phosphorylation regulates the cardiac ryanodine receptor. Circ Res. 2004;94(6):e61-e70.

70. Aimé-Sempé C, et al. Myocardial cell death in fibrillating and dilated human right atria. J Am Coll Cardiol. 1999;34(5):1577-1586.

71. Allessie M, Ausma J, Schotten U. Electrical, contractile and structural remodeling during atrial fibrillation. Cardiovasc Res. 2002;54(2):230-246.

72. Zhu WZ, et al. Linkage of beta1-adrenergic stimulation to apoptotic heart cell death through protein kinase A-independent activation of Ca2+/calmodulin kinase II.J Clin Invest. 2003;111(5):617-625.

73. Porter AG, Jänicke RU. Emerging roles of caspase-3 in apoptosis. Cell Death Differ. 1999;6(2):99-104.

74. Madamanchi NR, Runge MS. Redox signaling in cardiovascular health and disease. Free Radic Biol Med. 2013;61:473-501.

75. Xie W, et al. Mitochondrial oxidative stress promotes atrial fibrillation. Sci Rep. 2015;5:11427.

76. Zorov DB, Filburn CR, Klotz LO, Zweier JL, Sollott SJ. Reactive oxygen species (ROS)-induced ROS release: a new phenomenon accompanying induction of the mitochondrial permeability transition in cardiac myocytes. J Exp Med. 2000;192(7):1001-1014.

77. Selnick HG, et al. Discovery of MK-8719, a potent O-GlcNAcase inhibitor as a potential treatment for tauopathies. JMed Chem. 2019;62(22):10062-10097.

78. Abdel-Magid AF. Inhibition of O-GlcNAcase (OGA): a potential therapeutic target to treat Alzheimer's disease. ACS Med Chem Lett. 2014;5(12):1270-1271.

79. Yuzwa SA, et al. Pharmacological inhibition of O-GlcNAcase (OGA) prevents cognitive decline and amyloid plaque formation in bigenic tau/ APP mutant mice. Mol Neurodegener. 2014;9:42.

80. Ramirez-Correa GA, et al. O-linked GlcNAc modification of cardiac myofilament proteins: a novel regulator of myocardial contractile function. Circ Res. 2008;103(12):1354-1358.

81. Banerjee PS, Ma J, Hart GW. Diabetes-associated dysregulation of O-GlcNAcylation in rat cardiac mitochondria. Proc Natl Acad Sci US A. 2015;112(19):6050-6055.

82. Watson LJ, et al. O-linked $\beta$-N-acetylglucosamine transferase is indispensable in the failing heart. Proc Natl Acad Sci U S A. 2010;107(41):17797-17802.

83. Zinman B, et al. Empagliflozin, cardiovascular outcomes, and mortality in type 2 diabetes. $N$ EnglJ Med. 2015;373(22):2117-2128.

84. McMurray JJV, et al. Dapagliflozin in patients with heart failure and reduced ejection fraction. N Engl J Med. 2019;381(21):1995-2008.

85. Chen J, et al. Quantitative PCR tissue expression profiling of the human SGLT2 gene and related family members. Diabetes Ther. 2010;1(2):57-92.

86. Mustroph J, et al. Empagliflozin reduces $\mathrm{Ca} /$ calmodulin-dependent kinase II activity in isolated ventricular cardiomyocytes. ESC Heart Fail. 2018;5(4):642-648.

87. Zelniker TA, et al Effect of dapagliflozin on atria fibrillation in patients with type 2 diabetes mellitus: insights from the DECLARE-TIMI 58 trial. Circulation. 2020;141(15):1227-1234.

88. Joubert M, et al. The sodium-glucose cotransporter 2 inhibitor dapagliflozin prevents cardiomyopathy in a diabetic lipodystrophic mouse model. Diabetes. 2017;66(4):1030-1040.

89. Shao Q, et al. Empagliflozin, a sodium glucose co-transporter-2 inhibitor, alleviates atrial remodeling and improves mitochondrial function in high-fat diet/streptozotocin-induced diabetic rats. Cardiovasc Diabetol. 2019;18(1):165.

90. Trum M, Wagner S, Maier LS, Mustroph J. CaMKII and GLUT1 in heart failure and the role of gliflozins. Biochim Biophys Acta Mol Basis Dis. 2020;1866(6):165729.

91. Ozcan L, et al. Calcium signaling through CaMKII regulates hepatic glucose production in fasting and obesity. Cell Metab. 2012;15(5):739-751.

92. Ozcan L, Cristina de Souza J, Harari AA, Backs J, Olson EN, Tabas I. Activation of calcium/calmodulin-dependent protein kinase II in obesity mediates suppression of hepatic insulin signaling. Cell Metab. 2013;18(6):803-815.

93. Ozcan L, et al. Treatment of obese insulin-resistant mice with an allosteric MAPKAPK2/3 inhibitor lowers blood glucose and improves insulin sensitivity. Diabetes. 2015;64(10):3396-3405.

94. Voigt N, et al. Enhanced sarcoplasmic reticulum $\mathrm{Ca} 2+$ leak and increased $\mathrm{Na}+-\mathrm{Ca} 2+$ exchanger function underlie delayed afterdepolarizations in patients with chronic atrial fibrillation. Circulation. 2012;125(17):2059-2070. 